\title{
Which parameters are important? Differential Importance under Uncertainty
}

\author{
Isadora Antoniano-Villalobos, ${ }^{1,2}$ Emanuele Borgonovo ${ }^{1,2}$ Sumeda Siriwardena ${ }^{3}$ \\ This is the peer reviewed version of the following article: Antoniano-Villalobos, I., Borgonovo, \\ E. and Siriwardena, S. (2018), Which Parameters Are Important? Differential Importance \\ Under Uncertainty. Risk Analysis, 38: 2459-2477, which has been published in final form at \\ https://doi.org/10.1111/risa.13125. This article may be used for non-commercial \\ purposes in accordance with Wiley Terms and Conditions for Use of Self-Archived Versions.
}

\begin{abstract}
In probabilistic risk assessment, attention is often focused on the expected value of a risk metric. The sensitivity of this expectation to changes in the parameters of the distribution characterizing uncertainty in the inputs becomes of interest. Approaches based on differentiation encounter limitations when a) distributional parameters are expressed in different units or b) the analyst wishes to transfer sensitivity insights from individual parameters to parameter groups, when alternating between different levels of a probabilistic safety assessment model. Moreover, the analyst may also wish to examine the effect of assuming independence among inputs. This work proposes an approach based on the differential importance measure, which solves these issues. Estimation aspects are discussed in detail, in particular the problem of obtaining all sensitivity measures from a single Monte Carlo sample, thus avoiding potentially costly model runs. The approach is illustrated through an analytical example, highlighting how it can be used to assess the impact of removing the independence assumption. An application to the probabilistic risk assessment model of the Advanced Test Reactor large loss of coolant accident sequence, concludes the work.
\end{abstract}

KEY WORDS: risk analysis, importance measures, sensitivity analysis, uncertainty analysis

\section{INTRODUCTION}

Risk analysts often benefit from quantitative information (Apostolakis, 2004) generated by computer codes, specifically developed to shed light on

\footnotetext{
${ }^{1}$ Department of Decision Sciences, Bocconi University, Milan, Italy, isadora.antoniano@unibocconi.it

${ }^{2}$ Bocconi Institute for Data Science and Analytics (BIDSA), Bocconi University, Milan, Italy, emanuele.borgonovo@unibocconi.it

${ }^{3}$ Statistics Division (ESS), Food and Agriculture Organization of the United Nations, Rome, Italy, sumeda.siriwardena@fao.org
}

complex risk assessment problems. The use of uncertainty and global sensitivity analysis techniques has become part of best practices (Helton \& Davis, 2002; Saltelli, 2002). Applications of sensitivity analysis in probabilistic risk and safety assessment (PSA) are transversal, including nuclear risk assessment (Iman \& Helton, 1988; Iman \& Hora, 1990; Helton \& Davis, 2002), natural risk assessment (e.g. hurricane loss prediction Iman et al. 2005; or flood risk modelling Koks et al. 2015), health risk assessment (Duintjer Tebbens et al., 2008), and climate change modelling (Anderson et al., 2014), to name a few.

The analysis often has two main targets. The 
first target regards the identification of the model inputs that drive uncertainty in the model output, a notion formalized as factor prioritization (Saltelli \& Tarantola, 2002; Saltelli, 2002). In the words of Helton et al. (2006, p. 1175), "uncertainty analysis refers to the determination of the uncertainty in analysis results that derives from uncertainty in analysis inputs, sensitivity analysis refers to the determination of the contributions of individual uncertain analysis inputs to the uncertainty in analysis results". Factor prioritization is relevant for model development and implementation. In the model development phase, knowledge of the most important parameters helps to determine the parts of the model requiring a more detailed investigation, as well as those for which further information collection is more effective. In the implementation phase, knowledge of the most important parameters helps the analyst to identify the factors on which to focus managerial attention (Eschenbach, 1992).

The second target regards the identification of the trend (sign of change) of the decision criterion as a function of the model inputs. It is of interest whether an increase (decrease) in a given model input leads to an increase (decrease) in the model output. An important indication of trend is provided by the gradient of the decision criterion (see, among others, Tsanakas \& Millossovich, 2016). Indeed, partial derivatives are the key sensitivity measures in the comparative statics methodology of Samuelson (1941), a crucial approach to sensitivity analysis in the Economics literature. Partial derivatives are also a fundamental tool for sensitivity analysis in reliability and risk assessment, as discussed by Helton (1993) and Li et al. (2011) (see also Borgonovo \& Plischke, 2016, for a review of trend identification methods).

Under uncertainty, the expected value of a risk metric becomes the reference quantity in several investigations (Helton \& Breeding, 1993). The numerical value of such expectation depends on the values of the parameters that characterize the distribution of the model inputs, usually derived from data or expert opinions (Saltelli \& Tarantola, 2002). Lack or sparsity of the data may prevent the analyst from assigning a precise value to the parameters of the model inputs' distribution. As underlined, among others, by Rubinstein (1989) and Glasserman \& Liu (2010), it then becomes important to study the sensitivity of the expected value of the risk metric to changes in the distributional parameters, a task that can lead to important insights. Consider an analyst who wishes to use information provided by the partial derivatives of the risk metric in a probabilistic safety assessment (PSA) context. To illustrate, assume that the expectation of interest is the mean time to failure (MTTF) of a given system. The system is made of several components, joined by a given structure function. The failure times are described by independent Weibull distributions. Then, the parameters of the model inputs' distribution are the set of scale and shape parameters of each component's Weibull density. Using the likelihood ratio method (LRM), the analyst obtains estimates of the sensitivity of the MTTF to the distributional parameters (Davani, 1994). However, the units of the Weibull scale parameters are $1 /$ [time], while the shape parameters have no units. Thus, the partial derivatives of the system MTTF with respect to the scale parameters cannot be compared to their counterparts corresponding to the shape parameters: no conclusion on the ranking is possible ( $\mathrm{Li}$ et al., 2011). Moreover, if the system has $n$ components, there will be $n$ scale parameters and $n$ shape parameters. Even with shared units, it would not be possible to identify the most important component, because each component is associated with two derivatives. Thus, the ranking problem requires a natural way to aggregate sensitivities.

The present work proposes a solution to these issues by extending the differential importance measure of Borgonovo \& Apostolakis (2001) to a stochastic simulation context (an intuition previously mentioned by Li et al., 2011), obtaining what we call the stochastic differential (SD) importance measure. The differential importance measure is based on a rescaling of partial derivatives, combining the gradient information into a normalized differential. As a result, the sensitivity measure is both additive and unitless. Thus, the comparison of magnitudes of the sensitivity indices becomes meaningful even when the parameters have different units. Moreover, by additivity, the importance of a component (which can be represented by a collection of parameters) is recovered as the sum of the importance measures of the parameters involved, i.e., by the simplest aggregation rule.

This leads to discuss estimation in detail. We introduce and compare two estimators which allow the calculation of sensitivity measures for all parameters, directly from the input-output sample produced by a single Monte Carlo loop: a direct estimator and one based on importance sampling. The interpretation of results is focused, not only on sensitivity analysis 
settings, but also on the different levels of uncertainty at which the distributions are assigned. Analytical examples and a realistic size application, the large loss of coolant accident sequence of the ATR reactor, illustrate how the method can be used to assess the consequences of assuming independence of the model inputs in an uncertainty analysis. Results show that, as a consequence of the different parameterizations, notable differences in sensitivity analysis results emerge regarding the importance of parameters, as well as the direction of their impact on the expected value of the risk metric.

The remainder of the paper is organized as follows. Section 2 reviews some relevant literature and formalizes the notation. Section 3 extends the differential importance measure to models with stochastic output. Section 4 addresses estimation. Section 5 illustrates the approach for studying the effects of correlations. Section 6 discusses the interpretations of results and section 7 presents some results for a well known risk assessment application.

\section{RELATED LITERATURE}

Sensitivity analysis has become an integral part of scientific modelling (Saltelli et al., 2000). Therefore, the literature on sensitivity analysis is vast and a complete review cannot be comprised within the present paper. While this section reviews the methods more closely related to our work, we refer to Saltelli et al. (2005, 2012); Ghanem et al. (2016), and Borgonovo \& Plischke (2016) for broad overviews, and to Frey \& Patil (2002) and Helton \& Davis (2002) for reviews with a focus on risk analysis applications. In general, one can distinguish two main classes of methods, local and global (Helton, 1993). Local methods study the sensitivity of the model output to small variations of the model inputs around a base case. Among them, one-factor-at-a-time methods and one-way sensitivity functions (van der Gaag et al., 2007; Castillo et al., 1996, 1997), together with their graphical representations, namely, tornado diagrams (Howard, 1988) and spiderplots (Eschenbach, 1992), have become standard and are implemented in popular software tools. Partial derivatives stand out as one of the most popular methods, and Helton (1993) shows that the rationale for using them derives from the Taylor expansion of the model output with respect to small perturbations in the model inputs. Borgonovo (2008) extends this idea to the case in which the model inputs are constrained.
One of the limitations of any local sensitivity method is that it does not take into account uncertainty about the model inputs (Helton, 1993; Saltelli et al., 2004; Oakley \& O'Hagan, 2004). Conversely, global sensitivity methods have the purpose of allowing the analyst to appreciate the variability of the model output, while taking into account model input uncertainty. In a global sensitivity analysis the model inputs are assigned a support and, possibly, a joint distribution. The variability of the model output as the model inputs vary within their support is typically assessed through a Monte Carlo simulation, where the model inputs are either sampled through crude Monte Carlo or generated by some form of Latin-Hypercube and quasi-Monte Carlo sequence (Helton \& Davis, 2003). Some of the most popular global sensitivity analysis methods are non-parametric (or regression-based) methods (Helton, 1993; Helton et al., 2006; Storlie et al., 2009), variance-based methods (Iman \& Hora, 1990; Saltelli, 2002; Saltelli et al., 2008) and momentindependent methods (Borgonovo, 2006; Borgonovo \& Tarantola, 2008). We refer to Borgonovo et al. (2016) for a rationale that encompasses several global sensitivity measures. In non-parametric methods, the measures of sensitivity are the Pearson correlation coefficients or Standardized regression coefficients between the model output and individual model inputs (Storlie et al., 2009). In variance-based sensitivity analysis, a fundamental role is played by the functional ANOVA expansion of the model output (Efron \& Stein, 1981; Sobol', 1990), and the preferred sensitivity measures are the fractional contributions of the model inputs, individual or total (Saltelli \& Tarantola, 2002), to the variance of the model output. In a moment-independent sensitivity analysis, the sensitivity measures derive from some form of separation measure between the conditional and unconditional model output distributions, defined with respect to their densities or cumulative distribution functions (Borgonovo et al., 2016).

Efforts have been made to bridge the gap between local and global methods. Bier (1983) and Helton (1993) introduce partial derivatives multiplied by the standard deviation of the uncertain model inputs, as sensitivity measures. This follows from the variance of the first order Taylor expansion of the model (Helton, 1993). The derivative-based sensitivity measures of Sobol' \& Kucherenko (2009) and the Distributed Evaluation of Local Sensitivity Analysis (DELSA) method of Rakovec et al. (2014) 
are based on the idea of estimating local sensitivity at randomized locations in the model input space, and defining sensitivity measures in terms of the expected value of local sensitivities (see Lamboni et al., 2013; Roustant et al., 2014, for further details).

The techniques discussed so far have been mainly developed for cases in which the relationship between the model inputs and the model output is deterministic. However, in relevant risk analysis applications, the input-output mapping must be regarded as stochastic. Then, in several studies, the expected value of the model output becomes the target of the sensitivity analysis. In Queuing Theory, the customer's average waiting time is often analyzed (Whitt, 2006). In Reliability Theory, a common object of study is the mean time to failure which is, in fact, the expected value of the system random failure time (Davani, 1994). Hong (2009) focuses the sensitivity analysis on the probability that the stochastic model output falls below a given quantile. Financial and insurance analysts deal with stochastic losses and apply risk measures for risk management purposes (Artzner et al., 1999; Belles-Sampera et al., 2014). Popular risk measures are value-at-risk (value) and conditional-value-atrisk (see Artzner et al., 1999; Belles-Sampera et al., 2014, for additional details). When risk measures are the target of the sensitivity, one is considering the sensitivity of a functional of the model output. Tsanakas \& Millossovich (2016) introduce a novel approach to the sensitivity of risk measures, based on computing the partial derivatives of the risk measures after each model input of interest has undergone a random perturbation.

The literature on sensitivity analysis also distinguishes between different settings and it is important for the analyst to clarify the sensitivity analysis question being asked, so as to identify the most appropriate method to use (Saltelli et al., 2008). The extensive literature on the subject usually contemplates five sensitivity analysis settings: factor prioritization, factor fixing, trend indentification, interaction quantification and stability determination (relevant when the analyst is concerned about whether the change in the model inputs may alter the optimal policy). No single method can tackle these five settings simultaneously (Borgonovo \& Plischke, 2016). In this work, we focus on factor prioritization and trend identification for models whose response to the inputs is stochastic.

Because in the following sections we address the sensitivity of the expected value of a risk metric to the parameters of model input distributions, the results of Rubinstein (1989); Rubinstein \& Shapiro (1993); Glasserman \& Liu (2010) regarding the score function method are of particular relevance and will be discussed below in further detail.

\subsection{Differential Sensitivity Analysis: from model inputs to distribution parameters}

The traditional framework of sensitivity analysis, considers a simulation code (a model) that estimates $d$ quantities of interest as a function of $n$ model inputs. Denote the input-output mapping by $\mathbf{y}=$ $g(\mathbf{x})$, where $\mathbf{y}=\left\{y_{1}, y_{2}, \ldots, y_{d}\right\}$ is the model output vector, $\mathbf{x}=\left\{x_{1}, x_{2}, \ldots, x_{n}\right\}$ is the vector of model inputs and $g: \mathcal{X} \rightarrow \mathbb{R}^{d}$ is a deterministic multivariate function. The model input space, $\mathcal{X} \subseteq \mathbb{R}^{n}$, is a subset of the natural domain of $g$. For simplicity, in the remainder, we shall focus on $d=1$.

Partial derivatives are among the most popular sensitivity measures. The rationale is presented by Helton (1993), even if their use dates further back in time. Given a reference point $\mathbf{x}^{0}$, if the inputoutput mapping, $g$, is smooth and the changes in the inputs are sufficiently small, the change in model output, $\Delta g=g(\mathbf{x})-g\left(\mathbf{x}^{0}\right)$, can be approximated by the first order Taylor expansion, $\sum_{j=1}^{n} g_{i}^{\prime}\left(\mathbf{x}^{0}\right)\left(x_{i}-x_{i}^{0}\right)$, with a negligible error (further details in Appendix). Thus, the partial derivatives $g_{i}^{\prime}\left(\mathbf{x}^{0}\right)$ become natural measures of the sensitivity of $g$ to perturbations of the model inputs. The same idea justifies, for instance, the comparative statics technique of Samuelson (1941), in economics. Regarding sensitivity settings, partial derivatives provide information with respect to sign of change, as they indicate whether the perturbation in the model input leads to an increase or decrease in the model output. However, partial derivatives alone cannot be directly used for factor prioritization if the model inputs are denominated in different units. Therefore, alternative normalizations are proposed in the literature. A common practice is to multiply the partial derivative by the base case value of the model input, thus turning the partial derivative into a semi-elasticity.

A further limitation of the use of derivatives as sensitivity measures, is encountered when the model inputs are uncertain and the analyst is interested in exploring the sensitivity of $Y$ to variations of the model inputs in the complete support $\mathcal{X}$. In this case, one is implicitly considering a probability space $\left(\mathcal{X}, \mathcal{B}(\mathcal{X}), \mathbb{P}_{\mathbf{X}}\right)$, where $\mathbb{P}_{\mathbf{X}}$ denotes a joint probability 
measure over the space $\mathcal{X}$ of model inputs. It is usual to assume that $\mathbb{P}_{\mathbf{X}}$ has a joint parametric density function, $f(\mathbf{X} ; \boldsymbol{\theta})$, for some $\boldsymbol{\theta}=\left(\theta_{1}, \ldots, \theta_{m}\right)$. The model inputs $\mathbf{X}$ are therefore treated as random variables, and denoted with $\mathbf{X}$, while $\mathbf{x}$ denotes one of their possible realizations. Observe that, for a fixed value of $\theta$, one does not obtain a deterministic value of $Y$, but a distribution with density $f_{Y}(\cdot \mid \theta)$, induced by $f_{\mathbf{X}}(\cdot ; \boldsymbol{\theta})$ and the transformation $Y=g(X)$.

As previously discussed, in several applications the quantity of interest is the expected value or some other functional of $Y$. This impacts the way in which sensitivity analysis is performed. Specifically, one has a conditional stochastic behaviour $\mathbf{X} \mid \boldsymbol{\theta} \sim f_{\mathbf{X}}(\cdot ; \boldsymbol{\theta})$, where $\boldsymbol{\theta}$ denotes an $m$-dimensional vector of parameters characterizing the model input distribution. Let

$$
L(\boldsymbol{\theta})=\mathbb{E}[T(Y) ; \boldsymbol{\theta}]=\int_{\mathbf{X}} T(g(\mathbf{x})) f_{\mathbf{X}}(\mathbf{x} ; \boldsymbol{\theta}) \mathrm{d} \mathbf{x},
$$

for some transformation $T: \mathbb{R} \longmapsto \mathbb{R}$ not depending on $\theta$. For example, when $T$ is the identity, $L(\boldsymbol{\theta})=$ $\mathbb{E}[Y ; \boldsymbol{\theta}]$, and tail probabilities are recovered from indicator functions of the type $T(Y)=\mathbb{I}\{Y>y\}$ (Hong, 2009). Then, the sensitivity question involves the study of changes in $L(\boldsymbol{\theta})$ with respect to the parameters, $\theta$, of the model input distribution. In particular, Rubinstein (1989) considers a quantity of interest expressed as

$$
\varphi[L(\theta)]=\varphi\left[\int_{\mathcal{X}} T(g(\mathbf{x})) f_{\mathbf{X}}(\mathbf{x} ; \theta) \mathrm{d} \mathbf{x}\right]
$$

for some operator $\varphi$ acting on $L(\theta)$ through changes in $\theta$ only, such as a differentiation operator. If $\varphi$ commutes with the integration operator, then

$$
\varphi[L(\theta)]=\int_{\mathcal{X}} T(g(\mathbf{x})) \varphi\left[f_{\mathbf{X}}(\mathbf{x} ; \theta)\right] \mathrm{d} \mathbf{x} .
$$

In other words, $\varphi$ may be applied to the joint density of $\mathbf{X}$, before integrating over the model input space. The representation then becomes amenable to Monte Carlo estimation. Indeed, multiplying and dividing by $f_{\mathbf{X}}(\mathbf{x} ; \theta)$ leads to

$$
\begin{aligned}
\varphi[L(\boldsymbol{\theta})] & =\int_{\mathcal{X}} T(g(\mathbf{x})) \frac{\varphi\left[f_{\mathbf{X}}(x ; \theta)\right]}{f_{\mathbf{X}}(\mathbf{x} ; \theta)} f_{\mathbf{X}}(\mathbf{x} ; \theta) d x \\
& =\mathbb{E}\left[T(g(\mathbf{X})) \frac{\varphi\left[f_{\mathbf{X}}(\mathbf{X} ; \theta)\right]}{f_{\mathbf{X}}(\mathbf{X} ; \theta)}\right] .
\end{aligned}
$$

Our main interest here is in the derivative of the expected value of $Y$ with respect to each parameter $\theta_{i}$. Therefore, in the remainder and without loss of generality, $T$ will be the identity function and $\varphi$ will be the differentiation operator which, under regularity conditions, is known to commute with the integral. Therefore,

$$
L(\boldsymbol{\theta})=\mathbb{E}[Y ; \boldsymbol{\theta}]=\int_{\mathbf{X}} g(\mathbf{x}) f_{\mathbf{X}}(\mathbf{x} ; \boldsymbol{\theta}) \mathrm{d} \mathbf{x},
$$

and $\varphi(L(\theta))$ is again an expectation:

$$
\varphi[L(\theta)]=\nabla L(\theta)=\mathbb{E}[g(\mathbf{X}) \mathcal{S}(\theta ; \mathbf{X})] .
$$

The function $\mathcal{S}$, known as the score function of the distribution, is the gradient of the log-likelihood function, $\ln f_{\mathbf{X}}(\mathbf{x} ; \boldsymbol{\theta})$, with respect to $\boldsymbol{\theta} \cdot \varphi(L(\theta))$ is then an $m$-dimensional vector, with the $i$-th component given by

$$
\frac{\partial L(\theta)}{\partial \theta_{i}}=\int_{\mathcal{X}} g(\mathbf{x}) \mathcal{S}_{i}(\theta ; \mathbf{x}) f_{\mathbf{X}}(\mathbf{x} ; \theta) d \mathbf{x}=\mathbb{E}\left[g(\mathbf{X}) \mathcal{S}_{i}(\theta ; \mathbf{X})\right]
$$

where

$$
\mathcal{S}_{i}(\theta ; \mathbf{x})=\frac{\partial \ln f_{\mathbf{X}}(\mathbf{x} ; \theta)}{\partial \theta_{i}}=\frac{1}{f_{\mathbf{X}}(\mathbf{x} ; \theta)} \frac{\partial f_{\mathbf{X}}(\mathbf{x} ; \theta)}{\partial \theta_{i}} .
$$

It is, therefore, possible to obtain Monte Carlo estimators of the derivatives of $\mathbb{E}[Y ; \boldsymbol{\theta}]$ with respect to each $\theta_{i}$ and evaluated at parameter values $\boldsymbol{\theta}_{0}$, from a single sample of size $N$ from $f_{\mathbf{X}}\left(\cdot, \boldsymbol{\theta}_{0}\right)$ and the corresponding $N$ evaluations of $g(\mathbf{x})$. This idea, known as the score function method, has become popular for estimating the derivatives of some quantities of interest (see Li et al., 2011, among others). In risk analysis, this can be used to investigate the importance of parameters in the model input distribution. In particular, we propose to combine this approach with the differential importance measure, as discussed in the next section.

\section{A DIFFERENTIAL IMPORTANCE MEASURE FOR STOCHASTIC OUTPUT}

The advantage of using the differential importance measure instead of partial derivatives in a complex simulation setting has been underlined, for instance, by $\mathrm{Li}$ et al. (2011). In this section, we formalize the use of the differential importance measure of the expected value of a quantity of interest, with an emphasis on methodological aspects. For illustrative purposes, let us begin with an analytical example.

Example 1: Consider a parallel system, consisting of two different components. The quantity of interest is the random time to failure, $T=$ $\max \left(T_{1}, T_{2}\right)$, of the system, which depends on the 
failure times, $T_{1}$ and $T_{2}$, of the two components. Assuming that these follow independent Weibull distributions with cumulative distribution functions

$$
F_{i}(t)=1-e^{-\left(t / \lambda_{i}\right)^{\alpha_{i}}}, \quad i=1,2,
$$

the mean time to failure of the system is given by

$$
L(\boldsymbol{\theta})=\mathbb{E}[T]=\mathbb{E}\left[\max \left(T_{1}, T_{2}\right) ; \boldsymbol{\theta}\right],
$$

where $\boldsymbol{\theta}=\left(\lambda_{1}, \lambda_{2}, \alpha_{1}, \alpha_{2}\right)$ is the 4-dimensional vector of parameters of the joint distribution of $\left(T_{1}, T_{2}\right)$. In this case, all the relevant quantities can be computed analytically. Assuming, for instance, that the parameters have been estimated as $\lambda_{1}=1$, $\lambda_{2}=2, \alpha_{1}=1.3, \alpha_{2}=1.5$, then, $\mathbb{E}[T]=0.732$ and the sensitivities of $L(\boldsymbol{\theta})$ to the four parameters, measured by the partial derivatives, are:

$$
\begin{array}{rlrl}
\frac{\partial L}{\partial \lambda_{1}} & =0.521 ; & & \frac{\partial L}{\partial \alpha_{1}}=0.028 ; \\
\frac{\partial L}{\partial \lambda_{2}}=0.106 ; & \frac{\partial L}{\partial \alpha_{2}}=0.096 .
\end{array}
$$

These results may be interpreted with respect to the different questions of interest in sensitivity analysis. Regarding trend, we can surely conclude that an increase in each of the parameters leads to an increase in the mean time to failure of the system. Regarding factor prioritization, $\lambda_{1}$ is associated with the highest derivative. However, it would not be correct to state that $\lambda_{1}$ is the most important parameter, because its derivative cannot be compared with $\partial L / \partial \alpha_{1}$ or $\partial L / \partial \alpha_{2}$, which have different units. Nor it is possible to assess whether the parameters $\left(\lambda_{1}, \alpha_{1}\right)$ associated to the distribution of component 1 are more influential than those for component 2 , namely $\left(\lambda_{2}, \alpha_{2}\right)$. This is due to the fact that $\partial L / \partial \lambda_{1}>\partial L / \partial \lambda_{2}$, but $\partial L / \partial \alpha_{1}<\partial L / \partial \alpha_{2}$.

To overcome these limitations, we express the sensitivity measures as differential importance measures of the parameters (Borgonovo \& Apostolakis, 2001). In general, consider the value assumed by the performance measure $L(\boldsymbol{\theta})=\mathbb{E}[g(\boldsymbol{X}) ; \theta]$ and denote by $L_{i}^{\prime}(\boldsymbol{\theta})$ the partial derivative of $L(\boldsymbol{\theta})$ with respect to $\theta_{i}$, evaluated at $\boldsymbol{\theta}$, for $i=1, \ldots m$. Under the assumption that $\sum_{i=1}^{m} L_{i}^{\prime}(\boldsymbol{\theta}) \mathrm{d} \theta_{i} \neq 0$, we call the quantity

$$
\mathrm{SD}_{j}=\frac{L_{j}^{\prime}(\boldsymbol{\theta}) \mathrm{d} \theta_{j}}{\sum_{i=1}^{n} L_{i}^{\prime}(\boldsymbol{\theta}) \mathrm{d} \theta_{i}},
$$

the stochastic differential (SD) importance measure of the $j$-th distribution parameter, $\theta_{j}$, where $\mathrm{d} \theta_{j}$ denotes a small (infinitesimal) perturbation of the $j$-th parameter. The numerator in this expression represents the differential change in $L(\boldsymbol{\theta})$ due to a perturbation of $\theta_{j}$, while the denominator accounts for the differential change due to a change of all parameters (further details in Appendix). In this way, the importance of a parameter depends, not only on the value of the partial derivative, but also on the way in which parameters are perturbed relative to each other, providing the analyst with additional degrees of freedom. In the literature, two cases of perturbations have been given great attention: uniform perturbations, when $\mathrm{d} \theta_{i}=\mathrm{d} \theta_{j}$ for every $i, j$; proportional perturbations, when $\mathrm{d} \theta_{i} / \theta_{i}=\mathrm{d} \theta_{j} / \theta_{j}$ for every $i, j$. The corresponding SD measures, denoted by $\mathrm{uSD}$ and $\mathrm{pSD}$ in the remainder, are given by:

$$
\begin{aligned}
\text { (a) } \mathrm{uSD}_{j} & =\frac{L_{j}^{\prime}(\boldsymbol{\theta})}{\sum_{i=1}^{n} L_{i}^{\prime}(\boldsymbol{\theta})} ; \\
\text { (b) } \mathrm{pSD}_{j} & =\frac{L_{j}^{\prime}(\boldsymbol{\theta}) \theta_{j}}{\sum_{i=1}^{n} L_{j}^{\prime}(\boldsymbol{\theta}) \theta_{i}} .
\end{aligned}
$$

Equation (12-a) suggests that SD produces the same ranking as partial derivatives, under an assumption of uniform perturbations of the parameters. Similarly, equation (12-b) suggests that $\mathrm{SD}$ produces the same ranking as elasticities when proportional perturbations are applied. Indeed, the elasticity of parameter $j$ is given by $E_{j}=$ $L_{j}^{\prime}(\boldsymbol{\theta}) \theta_{j} / \mathbb{E}[g(\boldsymbol{X}) ; \theta]$ and it is enough to divide the numerator and denominator of equation (12-b) by $\mathbb{E}[g(\boldsymbol{X}) ; \theta]$ to obtain $\mathrm{pSD}_{j}=E_{j} / \sum_{i=1}^{n} E_{i}$.

It is useful to read these results in the converse direction. Ranking with partial derivatives is equivalent to assuming uniform perturbations of the parameters; ranking with elasticities is equivalent to assuming proportional perturbations. The conditions for applying each of the two assumptions depend on the model and the problem at hand. For instance, uniform perturbations are sensible only if the parameters have the same units.

Moreover, SD possesses the additivity property. Let $v=\left(i_{1}, i_{2}, \ldots, i_{k}\right)$ be a set of parameter indices. The differential of $\mathbb{E}[g(\boldsymbol{X}) ; \theta]$ when the corresponding group of parameters is perturbed is equal to

$\mathrm{d}_{v} \mathbb{E}[g(\boldsymbol{X}) ; \theta]=\frac{\partial \mathbb{E}[g(\boldsymbol{X}) ; \theta]}{\partial \theta_{i_{1}}} \mathrm{~d} \theta_{i_{1}}+\ldots+\frac{\partial \mathbb{E}[g(\boldsymbol{X}) ; \theta]}{\partial \theta_{i_{k}}} \mathrm{~d} \theta_{i_{k}}$,

so that

$\mathrm{SD}_{v}=\frac{L_{i_{1}}^{\prime}(\boldsymbol{\theta}) \mathrm{d} \theta_{i_{1}}+\ldots+L_{i_{k}}^{\prime}(\boldsymbol{\theta}) \mathrm{d} \theta_{i_{k}}}{\sum_{i=1}^{m} L_{i}^{\prime}(\boldsymbol{\theta}) \mathrm{d} \theta_{i}}=\mathrm{SD}_{i_{1}}+\ldots+\mathrm{SD}_{i_{k}}$. 
ExAmple 1 (continued): Considering proportional perturbations of the parameters, we obtain:

$$
\begin{aligned}
& \operatorname{pSD}_{\lambda_{1}}=0.57 ; \quad \operatorname{pSD}_{\alpha_{1}}=0.04 ; \\
& \operatorname{pSD}_{\lambda_{2}}=0.231 ; \quad \operatorname{pSD}_{\alpha_{2}}=0.158 .
\end{aligned}
$$

Clearly, $\lambda_{1}$ is the most important parameter, followed by $\lambda_{2}, \alpha_{2}$ and $\alpha_{1}$, in that order. By exploiting the additivity of SD, it is also possible to find the importance associated with each component, $T_{i}$, as the sum of the SD associated to the parameters of its marginal distribution:

$$
\begin{array}{ll}
\text { Component 1: } & \mathrm{pSD}_{T_{1}}=\mathrm{pSD}_{\lambda_{1}}+\mathrm{pSD}_{\alpha_{1}}=0.61 . \\
\text { Component 2: } & \mathrm{pSD}_{T_{2}}=\mathrm{pSD}_{\lambda_{2}}+\mathrm{pSD}_{\alpha_{2}}=0.389 .
\end{array}
$$

Thus, overall variations in the parameters of component 1 are more important than variations of the parameters of component 2. Similarly, the joint importances of the failure rates (scale parameters) and those of the shape parameters are, respectively, $\mathrm{pSD}_{\lambda_{1}}+\mathrm{pSD}_{\lambda_{2}}=0.801$ and $\mathrm{pSD}_{\alpha_{1}}+\mathrm{pSD}_{\alpha_{2}}=$ 0.199 , indicating that the scale parameters are more important than the shape parameters in driving the mean time to failure for our example.

Example 1 shows that, by exploiting the properties of the differential importance measure, it is straightforward to rank parameters, even when they have different units. Moreover, finding the joint importance of a group of parameters, i.e., those corresponding to a single marginal distribution, requires no additional effort. Thus, a practical way for solving the issues mentioned at the beginning of this section, is obtained.

\section{ESTIMATION}

Estimation of sensitivity measures is a delicate task, subject to the well known trade-off between estimation accuracy and computational efficiency. The greater the desired accuracy, the higher the number of model runs needed to achieve it. A large number of model runs makes the analysis infeasible, especially if the execution time of the model is high. We now illustrate how it is possible to obtain estimates of the stochastic differential importance measure from a single Monte Carlo sample. The advantage of a one-sample approach is to make the estimation independent of the number of model inputs. Under regularity conditions, which guarantee that $L_{j}^{\prime}(\boldsymbol{\theta})<\infty$, the Monte Carlo estimator of $L_{j}^{\prime}(\boldsymbol{\theta})$ is known to be consistent. Indeed, by the strong law of large numbers,

$$
\widehat{L_{j}^{\prime}(\boldsymbol{\theta})}=\lim _{N \rightarrow \infty} \frac{1}{N} \sum_{k=1}^{N} g\left(\mathbf{X}^{k}\right) \mathcal{S}_{j}\left(\theta ; \mathbf{X}^{k}\right) \underset{n \rightarrow \infty}{\stackrel{a . s}{\longrightarrow}} L_{j}^{\prime}(\boldsymbol{\theta}),
$$

where a.s. denotes almost sure convergence. The $\mathbf{X}^{k}, k=1, \ldots, N$ denote independent and identically distributed random variables from the joint input distribution with probability density function $f_{\mathbf{X}}(\cdot ; \boldsymbol{\theta})$, and $\mathcal{S}_{j}$ denotes the score function of equation (8). In this case, by Slutsky's theorem (Slutsky, 1925), the corresponding plug-in estimator of $\mathrm{SD}_{j}$ is weakly consistent. Formally,

$$
\widehat{\mathrm{SD}}_{j}=\widehat{\mathrm{SD}_{j}(\boldsymbol{\theta})}=\frac{\widehat{L_{j}^{\prime}(\boldsymbol{\theta})}}{\sum_{i=1}^{m} \widehat{L_{i}^{\prime}(\boldsymbol{\theta})}} \frac{\mathbb{P}}{n \rightarrow \infty} \frac{L_{j}^{\prime}(\boldsymbol{\theta})}{\sum_{i=1}^{m} L_{i}^{\prime}(\boldsymbol{\theta})}=\mathrm{SD}_{j} .
$$

Because the same Monte Carlo sample can be used to calculate SD estimates for all the $\theta_{j}$ parameters, the evaluation of the sensitivity measures of all distribution parameters can be achieved with a computational cost of $N$ model runs.

It is also possible to estimate SD for alternative values of $\boldsymbol{\theta}$, from the same $N$ model runs, by using importance sampling (see e.g. Rubinstein, 1989). Consider a value, $\boldsymbol{\theta}_{0}$, in the parameter space of the joint distribution of $\mathbf{X}$. The importance sampling ratio, $\Lambda\left(\boldsymbol{\theta}, \boldsymbol{\theta}_{0} ; \mathbf{x}\right)=f_{\mathbf{X}}(\mathbf{x} ; \boldsymbol{\theta}) / f_{\mathbf{X}}\left(\mathbf{x} ; \boldsymbol{\theta}_{0}\right)$, is well defined for every $\boldsymbol{\theta}$ for which $f_{\mathbf{X}}(\cdot ; \boldsymbol{\theta})$ is absolutely continuous with respect to $f_{\mathbf{X}}\left(\cdot ; \boldsymbol{\theta}_{0}\right)$. Then, for any such parameter value, one can write

$$
\begin{aligned}
L_{j}^{\prime}(\boldsymbol{\theta}) & =\mathbb{E}\left[g(\mathbf{X}) \mathcal{S}_{j}(\boldsymbol{\theta} ; \mathbf{X}) ; \boldsymbol{\theta}\right] \\
& =\mathbb{E}\left[g(\mathbf{X}) \mathcal{S}_{j}(\boldsymbol{\theta} ; \mathbf{X}) \Lambda\left(\boldsymbol{\theta}, \boldsymbol{\theta}_{0} ; \mathbf{X}\right) ; \boldsymbol{\theta}_{0}\right]
\end{aligned}
$$

Note that the expectation on the left side of this expression is taken with respect to $f_{\mathbf{X}}(\cdot ; \boldsymbol{\theta})$, while the expectation on the right is with respect to $f_{\mathbf{X}}\left(\cdot ; \boldsymbol{\theta}_{0}\right)$. This allows the estimation of $L_{j}^{\prime}(\boldsymbol{\theta})$ and, consequently, of $\mathrm{SD}_{j}(\boldsymbol{\theta})$ by:

$$
\begin{aligned}
{\widetilde{\mathrm{SD}_{j}}}_{j} & =\widetilde{\mathrm{SD}_{j}(\boldsymbol{\theta})} \\
& =\frac{\sum_{k=1}^{N} g\left(\widetilde{\mathbf{X}}^{k}\right) \mathcal{S}_{j}\left(\theta ; \widetilde{\mathbf{X}}^{k}\right) \Lambda\left(\boldsymbol{\theta}, \boldsymbol{\theta}_{0} ; \widetilde{\mathbf{X}}^{k}\right) \mathrm{d} \theta_{j}}{\sum_{i=1}^{m} \sum_{k=1}^{N} g\left(\widetilde{\mathbf{X}}^{k}\right) \mathcal{S}_{i}\left(\theta ; \widetilde{\mathbf{X}}^{k}\right) \Lambda\left(\boldsymbol{\theta}, \boldsymbol{\theta}_{0} ; \widetilde{\mathbf{X}}^{k}\right) \mathrm{d} \theta_{i}},
\end{aligned}
$$

where, for any $\boldsymbol{\theta}$, the $\widetilde{\mathbf{X}}^{k}$ are now sampled from a single joint density, $f_{\mathbf{X}}\left(\cdot ; \boldsymbol{\theta}_{0}\right)$. While this Importance Sampling estimator of is also weakly consistent for 
every value of $\boldsymbol{\theta}$ for which the importance sampling ratio is well defined, the convergence rate depends on the choice of $\boldsymbol{\theta}_{0}$ and, in general, one expects slower convergence compared to $\widehat{\mathrm{SD}}$. However, $\widetilde{\mathrm{SD}}$ has the great advantage of allowing the estimation of the complete surface $\left\{\mathrm{SD}_{j}(\theta): \theta \in \Theta, j=1, \ldots m\right\}$, with the same computational cost of $N$ model runs, at least in the most common case, when the support of $f_{\mathbf{X}}(\cdot ; \boldsymbol{\theta})$ does not depend on the parameters.

\section{FROM PARAMETERS TO MODEL INPUTS AND THE IMPACT OF CORRELATIONS}

One of the most important decisions when assigning distributions in sensitivity analysis studies, is whether or not to adopt an assumption of independence between model inputs. In this section, we discuss the use of SD for evaluating the effect of neglecting correlations and, at the same time, for determining the most important parameters (and, possibly, model inputs) in the presence of correlations. For simplicity of illustration, we consider a toy model for which analytical expressions are at reach.

Let $Y=g(\mathbf{X})=X_{1} X_{2}+X_{3}$, where $\mathbf{X}$ follows a three-variate normal distribution with mean $\mu$ and covariance matrix $\Sigma$, given by

$\mu=\left[\begin{array}{l}\mu_{1} \\ \mu_{2} \\ \mu_{3}\end{array}\right], \Sigma=\left[\begin{array}{ccc}\sigma_{1}^{2} & \rho_{1,2} \sigma_{1} \sigma_{2} & \rho_{1,3} \sigma_{1} \sigma_{3} \\ \rho_{1,2} \sigma_{1} \sigma_{2} & \sigma_{2}^{2} & \rho_{2,3} \sigma_{2} \sigma_{3} \\ \rho_{1,3} \sigma_{1} \sigma_{3} & \rho_{2,3} \sigma_{2} \sigma_{3} & \sigma_{3}^{2}\end{array}\right]$.

Assume that the quantity of interest is $L(\boldsymbol{\theta})=$ $\mathbb{E}[g(\mathbf{X}) ; \theta]=\mu_{1} \mu_{2}+\mu_{3}+\rho_{1,2} \sigma_{1} \sigma_{2}$, and the decision maker wishes to evaluate sensitivity with respect to the nine distribution parameters $\theta=\left(\mu, \sigma^{2}, \boldsymbol{\rho}\right)$, where $\mu$ and $\sigma^{2}$ represent the mean and variance of the marginal distributions of the model inputs, while their dependence structure is characterized by the pairwise correlation coefficients, $\boldsymbol{\rho}$. In this case, analytical expressions for pSD can be easily obtained by simple differentiation (see Appendix for details).

It is interesting to observe that, if independence between model inputs was assumed by the analyst, the joint distribution of $X$ would become a product of three univariate normal distributions, and $\boldsymbol{\theta}$ would be made up of only six parameters: the three means and the three variances. A direct analysis of the sensitivity of the model with respect to deviations from independence would not be possible. On the other hand, consider the full nine-parameter joint distribution. Sensitivity to deviations from independence can be assessed by looking at the sensitivity measures of each $\rho_{i, j}$ around zero. Note that parameters $\rho_{1,3}$ and $\rho_{2,3}$ have null importance, since $L(\boldsymbol{\theta})$ does not depend on them. That is, when studying the expected value of $Y$, determining the value of the correlation between $X_{3}$ and the other two model inputs, is not relevant: assuming independence between $\left(X_{1}, X_{2}\right)$ and $X_{3}$ has no consequences on the analysis. On the other hand, whenever $\sigma_{1}^{2}$ and $\sigma_{2}^{2}$ are strictly positive (for non-degenerate distributions), $\operatorname{pSD}_{\rho_{1,2}}>0$ even for $\rho_{1,2}=0$, so deviations from independence between $X_{1}$ and $X_{2}$ have a positive impact on $Y$. Depending on the magnitude of such impact, the analyst may decide whether it is reasonable to assume independence to simplify calculations or interpretation of other quantities, or whether further investigation is required in order to assess a possible dependence between the two model inputs.

Similarly, the variance, $\sigma_{3}^{2}$, of the third model input, has null importance; but the same does not hold for the remaining parameters. Figure 1 shows the values of $\mathrm{pSD}$ for each of the 9 distribution parameters, at two different sets of marginal parameter values, and with varying correlations $\rho_{1,2} \in[0,1]$. The marginal distribution of each $X_{i}$ is a standard normal on graph (a), while on graph (b), $\mu_{i}=1.1$ and $\sigma_{i}^{2}=0.25$. In other words, for example, plot (a) can be used to assess the sensitivity of the model to perturbations around parameter values $\mu=(0,0,0)$, $\sigma^{2}=(1,1,1)$ and all possible values of $\rho_{1,2}$. More specifically, for $\rho_{1,2}=0.5$ (on the horizontal axis) the mean of the third input has an importance measure of $p S D_{\mu_{3}}=2 / 3$ (in blue); the variances of the first and second components have equal importance $p S D_{\sigma_{1}^{2}}=p S D_{\sigma_{2}^{2}}=1 / 6$ (in light blue and green, respectively); for the correlation between the first and second components one registers $p S D_{\rho_{1,2}}=2 / 3$ (in mustard); all other parameters, at this point in the parameter space, have null importance.

Regarding factor prioritization, figure 1 shows that the mean parameters, $\mu_{1}$ and $\mu_{2}$, have null importance for the first parameter specification (a), and attention should be focused on the values of $\rho_{1,2}$ and $\mu_{3}$. On the other hand, for the second parameter specification (b), $\mu_{1}, \mu_{2}$ and $\mu_{3}$ have $p S D$ 's of similar magnitudes, consistently higher than those of $\sigma_{1}^{2}, \sigma_{2}^{2}$ and $\rho_{1,2}$, so that attention should be focused on the mean parameters if the analyst thinks this scenario better represents the parameter values of interest.

Regarding trend, we note that perturbations which increase $\rho_{1,2}$ or any of the mean parameters, lead to an increase in $\mathbb{E}[Y]$ for all of the parameter configurations considered. However, the increases in 

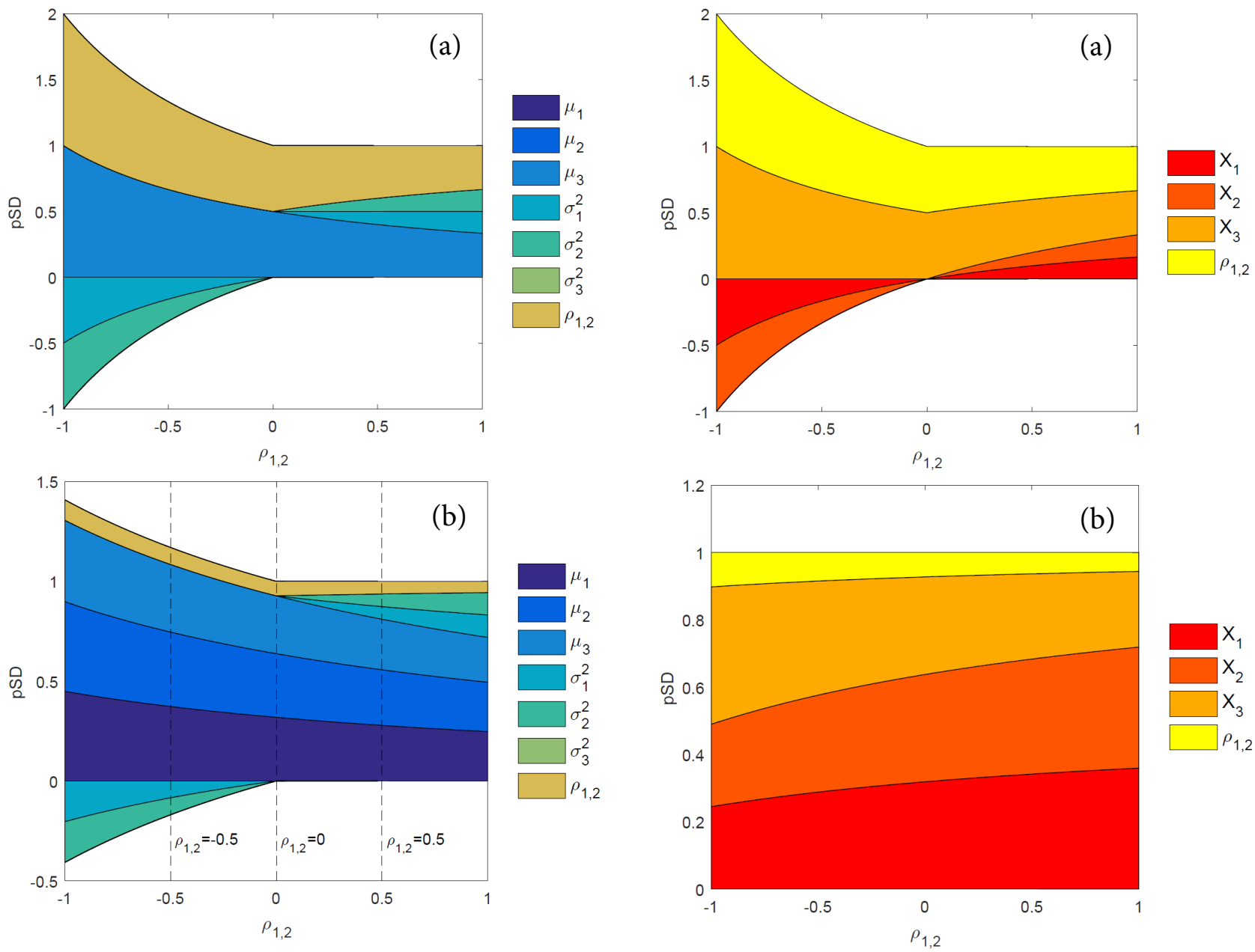

Fig. 1. pSD of individual parameters for the model $g(\mathbf{X})=$ $X_{1} X_{2}+X_{3}$ with tri-variate normal input, $\mathbf{X}$, with standard normal marginals (a) and $\mathrm{N}(1.1,0.25)$ marginals (b); $\rho_{1,2}$ varies in $[0,1]$ and all other correlations are irrelevant.

any of the two variances $\sigma_{1}^{2}$ and $\sigma_{2}^{2}$ have negative impacts on $\mathbb{E}[Y]$ for points in the parameter space with $\rho_{1,2}<0$, but this effect reverses for $\rho_{1,2}>0$.

The additivity of SD enables the evaluation of the importance of each $X_{i}$, identifying it with the aggregated contribution of all parameters involved in its marginal distribution. Similarly, we can isolate the importance of the dependence between model inputs by aggregating the SD measures of $\boldsymbol{\rho}$, which characterize the dependence structure. In fact, we can write $p S D_{X_{i}}=\operatorname{pSD}_{\mu_{i}}+\mathrm{pSD}_{\sigma_{i}^{2}}$ to denote the importance of the parameters of the marginal distribution of $X_{i}$, and let $p S D_{d e p}=\operatorname{pSD}_{\rho_{1,2}}+$ $\operatorname{pSD}_{\rho_{1,3}}+\operatorname{pSD}_{\rho_{2,3}}$ indicate the importance of the dependence structure. Note that, for this particular model, $p S D_{\text {dep }}=p S D_{\rho_{1,2}}$. These measures, at

Fig. 2. Joint contributions, $\mathrm{pSD}_{X_{i}}$, of the marginal parameters for each model input, and of the dependence structure, characterized by $\rho_{1,2}$, for the model and specifications of Figure 1.

the same parameter values discussed above, can be visualized in figure 2. Regarding factor prioritization, for the first set of parameter values (a) attention should be focused, with equal importance, on the parameters of the marginal distribution of $X_{3}$ and the dependence parameter $\rho_{1,2}$. However, in the second setting (b), the marginal parameters of $X_{1}$ and $X_{2}$ become more important than those of $X_{3}$, specially for values of $\rho_{1,2}$ close to 1 , while $\rho_{1,2}$ becomes the least relevant parameter. Regarding trend, for the first set of parameter values (a), and negative values of $\rho_{1,2}$, the perturbations in the parameters of $X_{1}$ and $X_{2}$ have an opposite effect on $\mathbb{E}[Y]$, while for $\rho_{1,2}>0$, all perturbations have increasing effects on $\mathbb{E}[Y]$. In the second scenario (b), all parameters have a positive relation with $\mathbb{E}[Y]$. 


\subsection{Numerical Estimation}

In the previous subsection, we have profited from the availability of analytical expressions. However, in the majority of real life situations, the input-output mapping, $g$, is known only through a computer code. The analyst, then, estimates the sensitivity measures numerically. The differential importance measure has been used in connection with score functions, for instance, in the last section of Li et al. (2011), but a thorough discussion of the estimation of $S D_{j}$ is missing. In this subsection, we discuss the numerical aspects of the analysis. We compare two strategies that involve, respectively, the use of a single sample in association with importance sampling, or the use multiple samples in association with Monte Carlo estimation. Details on the calculation of the score function for each of the parameters of interest can be found in Appendix.

Numerical experiments are performed for the second set of distribution parameters described above, with $\rho_{1,2} \in\{-0.5,0,0.5\}$. The corresponding analytical values of the sensitivity measures can be seen in graph (b) of figure 1, marked by three vertical dashed lines. The rationale for using these values of the correlation coefficient to illustrate the numerical estimation of pSD is to comment, at the same time, on the influence of this correlation coefficient on the convergence of the estimators, as the Monte Carlo sample size increases. For simplicity, the values of $\rho_{1,3}$ and $\rho_{2,3}$ are fixed at 0 throughout. Note that simple Monte Carlo estimation requires the evaluation of $g$ with three alternative samples, generated from three trivariate distributions with the same marginals, but three different values of $\rho_{1,2}$. In a real life application, repeated evaluations of the model may be too costly, and we may need to use a single sample to evaluate sensitivities at different points in the parameter space. An importance sampling estimator (equation 20) requires the evaluation of $g$ on a single sample, saving in terms of computational effort. Let $\tilde{\mathbf{X}} \sim \mathrm{N}\left(\mu_{0}, \Sigma_{0}\right)$ denote a three-variate normal random variable with mean $\mu_{0}$ and covariance matrix $\Sigma_{0}$. Then, the importance sampling ratio $f(\cdot, \boldsymbol{\theta})$ and $f\left(\cdot, \boldsymbol{\theta}_{0}\right)$ is always well defined:

$$
\begin{aligned}
& \Lambda\left(\boldsymbol{\theta}, \boldsymbol{\theta}_{0}, \tilde{\mathbf{x}}\right)=\frac{\left|\Sigma_{0}\right|}{|\Sigma|} \\
\times & \exp \left\{-\frac{(\tilde{\mathbf{x}}-\mu)^{\prime} \Sigma^{-1}(\tilde{\mathbf{x}}-\mu)-\left(\tilde{\mathbf{x}}-\mu_{0}\right)^{\prime} \Sigma_{0}^{-1}(\tilde{\mathbf{x}}-\mu)}{2}\right\},
\end{aligned}
$$

In this example, $\tilde{\mathbf{X}}$ is a vector of independent and identically distributed standard normal random variables, so that $\mu_{0}$ is the zero vector and $\Sigma_{0}$ is the identity matrix.

For each of the scenarios considered, estimates were calculated and boxplots were produced using Bootstraps samples of size $N_{B}=1000$, incurring a computational cost of $3 N$ model runs in the case of the Monte Carlo estimates, and only $N$ for the importance sampling estimates. Results are shown in Figure 3 for three parameters (rows), and the three values of $\rho_{1,2}$ considered (columns). Monte Carlo estimates, $\widehat{\mathrm{SD}}$, obtained from three different samples are shown in red; importance sampling estimates, $\widetilde{\mathrm{SD}}$, obtained from a single sample from a trivariate (independent) standard normal distribution, in blue. The black horizontal lines represent the true (analytic) values of SD. We observe that the convergence of the estimates as the sample size $N$ increases, is not affected by the value of the correlation coefficient. As expected, convergence is faster for the Monte Carlo estimators than for the importance sampling estimators, systematically, for all sensitivity measures. These results suggest that, if one wishes to use importance sampling with a single sample, the sample size should be the largest allowed by computational time.

\section{RESULT INTERPRETATION AND DISCUSSION}

In a probabilistic risk assessment context, the distinction between aleatory and epistemic uncertainty is relevant for result interpretation. This section considers how the interpretation of sensitivity measures changes, according to the type of uncertainty represented by the distribution parameters. Interested readers are referred to the works of Aven (2010, 2012, 2016); North (2010, 2011); Helton \& Sallaberry (2012); Flage et al. (2013) and Smith \& Gans (2015) for an overview of the treatment of uncertainties in risk analysis. Following Apostolakis (1990, 1994), we consider the model of the world, where the term world is used to denote the object of interest in the analysis. In other words, we write the output, $Y=g(\mathbf{X} \mid M, H)$, as a function of the vector, $\mathbf{X}$, of model inputs, the set, $M$, of model assumptions and the body of knowledge of the modeller, denoted by $H$. To fix ideas, recall example 1 . The quantity of interest is the MTTF and the model of the world is equation (9). Because the value of the MTTF depends on the parameters $\left(\lambda_{1}, \lambda_{2}, \alpha_{1}, \alpha, 2\right)$, equation (9) is a conditional model of the world (Apostolakis, 

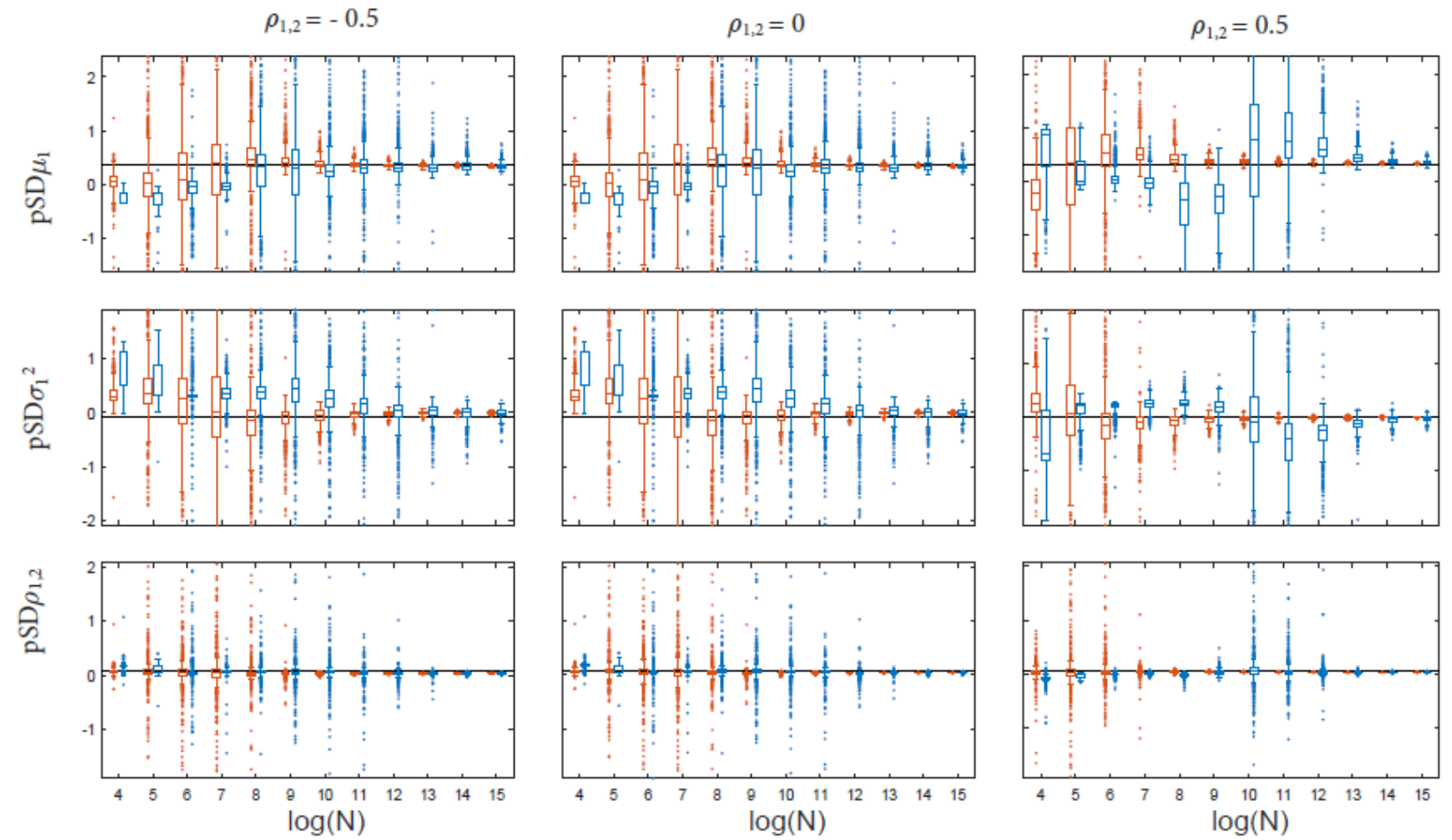

Fig. 3. Bootstrap box-plots of Montecarlo estimates $\widehat{\mathrm{pSD}}$ (red) and Importance Sampling estimates $\widetilde{\mathrm{pSD}}$ (blue) for parameters $\mu_{1}, \sigma_{1}^{2}$ and $\rho_{1,2}$. Three points in the parameter space are considered, corresponding to the vertical segments of figure 1 (b), where $\mu_{i}=1.1, \sigma_{i}^{2}=0.25, \rho_{1,3}=\rho_{2,3}=0$ and $\rho_{1,2} \in\{-0.5,0,0.5\}$. As the sample size $N$ increases, both estimates can be seen to converge to the true values (black), and the convergence is faster for $\widehat{\mathrm{pSD}}$.

1990). At this stage, an analyst is addressing the sensitivity of the output of the conditional model of the world, to the parameters of the aleatory distribution.

Considering perturbations to the parameters of the conditional model of the world, inherently implies uncertainty about their values: if there was certainty about the parameter values, there would be no need to make them vary in a sensitivity analysis. Uncertainty about parameters of the aleatory distribution is commonly known as epistemic uncertainty. There is an open debate about whether a probabilistic representation of uncertainty is exhaustive in the presence of deep uncertainties (Paté-Cornell, 1996; Helton \& Oberkampf, 2004; Pedroni et al., 2017). For instance, Aven \& Zio (2014, p. 1169) write "there is a need for developing broader frameworks where we see beyond probability to measure uncertainty." Such debate cannot be comprised within the present work, where it is assumed that available data and strength of knowledge allow the assignment of a distribution (either prior or posterior) to the parameters of the aleatory distribution. We call this the epistemic distribution and denote it by $\pi_{\boldsymbol{\Theta}}(\cdot ; \boldsymbol{\beta})$, where the capital letter $\boldsymbol{\Theta}$ indicates the fact that the vector $\boldsymbol{\theta}$ of parameters is now considered random.

To obtain the unconditional solution of the model of the world, following Apostolakis (1990, p. 1360), one averages the conditional model of the world over the possible values of the parameters. Going back to example 1 , the unconditional MTTF, denoted by $\mathcal{M T} \mathcal{T} \mathcal{F}$, can be derived as

$$
\begin{aligned}
\mathcal{L}(\beta)=\mathcal{M} \mathcal{T} \mathcal{T} \mathcal{F} & =\mathbb{E}_{\Theta}\left[\mathbb{E}_{\mathbf{T}}[T ; \theta] ; \beta\right] \\
& =\int_{\Theta} \mathbb{E}_{\mathbf{T}}[T ; \theta] \pi_{\boldsymbol{\Theta}}(\theta ; \beta) d \theta .
\end{aligned}
$$

Note that this expression is analogous to the $L(\boldsymbol{\theta})$ of equation (9), with the parameters of the epistemic distribution $(\boldsymbol{\beta})$ substituting those of the aleatory distribution $(\boldsymbol{\theta})$. The sensitivity indices now yield information about the effect of perturbations to the parameters of the epistemic distribution.

The method proposed here is part of a family of methods that consider perturbation analysis on functionals of a stochastic model output. Tsanakas \& Millossovich (2016) argue that these methods are 
global, quantitative and model free. Specifically, if the functional of interest involves an uncertainty quantification, then, the methods inspect the model on the entire model input space, $\mathcal{X}$, and are, therefore, global with respect to the model inputs. On the other hand, the analysis is conducted applying local perturbations on $\boldsymbol{\theta}$ and is, therefore, local in this respect. So far, we are not aware of studies applying global sensitivity methods to distributional parameters, although the work of Marrel et al. (2012) may be a first in this direction.

It is important to emphasize that the application of the method proposed here requires a number of choices on the part of the analyst. Recall (section 3 ) that the definition of SD accommodates any form of parameter perturbation. Thus, a first choice corresponds to the type of perturbation used. Uniform perturbations (which are equivalent to using partial derivatives) are sensible only when the parameters have the same units; while proportional perturbations are an intuitive solution for the case of parameters with different units. However, the general definition of SD, in equation (11), allows the analyst to study any specific set of relative variations. A second choice regards the parametrization of the distributions. For instance, in the case of a normally distributed model input, the analyst may choose between the variance or the standard deviation. This choice influences the shape of the function $L(\boldsymbol{\theta})$ and, therefore, may impact the results of the sensitivity analysis. Observe that the choice of the distribution parameters is common to any method addressing the sensitivity of a functional of the model output with respect to the parameters of the model input distributions. If some estimation method is used to find the parameter values of interest, it would be intuitive to choose the same parametrization for the sensitivity analysis.

The fact that the methods discussed here require an uncertainty quantification exposes them to a limitation, common to all global sensitivity analysis methods. In a global sensitivity analysis, "we assume to have information about the factors' probability distribution, either joint or marginal, with or without correlation, and that this knowledge comes from measurements, estimates, expert opinion, physical bounds, output from simulations, analogy with factors for similar species, and so forth" (Saltelli \& Tarantola, 2002). The assignment of the model input distribution is a delicate task. In some situations, lack of data may prevent the analyst from finding a unique parametric family that fits the data.
Alternatively, a mixture of parametric families may be assigned, or a non-parametric approach can be adopted. This is also a motivation for the present approach and, in general, any method focusing on the sensitivity of $L(\boldsymbol{\theta})$ : to appreciate the sensitivity of results on the given parameterization. However, if the model input distribution cannot be expressed through a single parametric form (or as a mixture of parametric distributions), then the method we propose is not applicable. Sensitivity analysis in the absence of a parametric distribution is an open research subject, as recent works (Confalonieri et al., 2010; Borgonovo et al., 2017) demonstrate.

\section{PROBABILISTIC SAFETY ASSESSMENT OF THE ATR REACTOR}

In this section, the large loss of coolant accident (LLOCA) of the advanced test reactor (ATR) is analyzed. The ATR is a nuclear reactor located at the Idaho National Laboratories. This model has become a classical test case, and several sensitivity measures have been computed for it. Probabilistic Safety Assessment (PSA) is the core technology used to conduct risk analysis of complex technological systems. The creation of a PSA model follows the risk analysis setup of the Rasmussen report (Nuclear Regulatory Commission, 1975) (see also Kaplan \& Garrick, 1981; Kaplan et al., 2001; Breeding et al., 1992; Helton \& Breeding, 1993; Apostolakis, 2004). The aim is to determine the undesired scenarios that can occur, their likelihood, and the corresponding consequences. In addition, PSA can produce indirect information, such as the importance of individual risk contributors. In the case of nuclear reactors, the PSA model is built to estimate a risk metric associated with one or more safety goals (Helton \& Breeding, 1993). Accurate reviews of the calculation of risk metrics in nuclear risk assessment can be found in the literature (Breeding et al., 1992; Helton \& Breeding, 1993; Smith, 1998). In the following discussion, the risk metric of interest is the core damage frequency (CDF).

The first step in a PSA is to identify a top event and trace out the individual hazards that could lead to it. The response of the plant to each group of Initiating Events (IE) is usually modelled via Event Trees (ET), which provide sequences that, depending on successes or failures of relevant systems, lead either to a safe or to a core damage state. In the case of the ATR-LLOCA sequence model, there is 
one initiating event, 44 basic events and 239 minimal cut sets (MCS). The model considers both aleatory and epistemic uncertainty. Aleatory uncertainty is expressed through basic event probabilities that quantify the likelihood of core damage. Uncertainty in the basic event probabilities is expressed by assigning an epistemic distribution, either directly to a basic event probability or to the failure rates of the corresponding exponential probability model. We call these the model inputs. As a consequence of epistemic uncertainty, the CDF becomes a random variable. The performance metric in this case study is the expected $\mathrm{CDF}, \mathbb{E}[C D F]$. The model has thirtyone inputs that correspond to either basic event probabilities or to failure rates of individual components. For example, the probabilities of basic events $8,14,19,21,26,28,32$ and 34 are expressed through exponential distributions and their corresponding failure rates are model inputs. For the list of all model inputs we refer to Table 1 of Borgonovo et al. (2003, p. 180).

The 31 inputs, $X_{i}$, are usually assigned independent log-normal distributions with parameters $\beta=\left(\mu_{i}, \sigma_{i}^{2}\right)_{i=1}^{31}$, corresponding to the mean and the variance of $\ln X_{i}$, respectively (equivalently, $\left.\ln X_{i} \sim \mathrm{N}\left(\mu_{i}, \sigma_{i}^{2}\right)\right)$. As discussed in section 5, sensitivity with respect to deviations from independence can be assessed directly by considering, instead, a joint distribution with 527 parameters. The full joint distribution adds to the marginal means and variances, all the parameters controlling the pairwise correlations between model inputs, $\rho_{i, j}=\operatorname{Cov}\left(\ln X_{i}, \ln X_{j}\right) / \sigma_{i} \sigma_{j}$. Assigning log-normal distributions to express epistemic uncertainty is quite common in probabilistic risk assessment. Thus, this discussion has a broad applicability outside the present case study. Some relevant details are presented in Appendix.

Because the distribution parameters have different units, the use of pSD (equation 12-b) is suggested. Monte Carlo estimates (equation (18) with $N=32,000)$ of the pSD importance of each of the 52 distribution parameters under the independence assumption, are shown in Figure 4. Perturbations are considered with respect to the same point in the parameter spaced studied in previous analysis. Specifically, the error factors, $\mathrm{EF}\left(X_{i}\right)=\exp \left\{1.645 \sigma_{i}\right\}$, are equal to 10 for all model inputs and the medians, $q_{2}\left(X_{i}\right)=\exp \left\{\mu_{i}\right\}$, are the same as reported in Table 1 of Borgonovo et al. (2003). Regarding factor prioritization, the mean parameters are the only important ones. That is, if independence is assumed, changes in the marginal variance parameters are negligible. Thus, if the analyst assumes independence at the epistemic uncertainty level of the ATR Large LOCA sequence, then attention needs to be focused on estimating the mean values of $\ln X_{i}$, and little attention need be paid to variances. Figure 6 shows bootstrap box-plots $\left(N_{B}=1,000\right)$ of $\widehat{\mathrm{pSD}}$ for the four most important parameters, $\mu_{17}, \mu_{27}, \mu_{18}, \mu_{3}$, for increasing $N$. Notice that, even for a relatively small Monte Carlo sample, $N=32$, the box plots are non-overlapping and the ranking of the parameters does not vary. Regarding trend identification, figure 4 shows that increases in any of the mean parameters lead to an increase in the expected core damage frequency. In fact, the estimated values of $\mathrm{pSD}_{\sigma_{i}}$ are negligible with respect to $\mathrm{pSD}_{\mu_{i}}$, but they all have a positive sign.

We now consider a second scenario, in which the analyst wishes to take into account a possible dependence structure between the model inputs, by choosing a multivariate log-normal distribution to characterize epistemic uncertainty (see equation (A.7) in Appendix) . Monte Carlo estimates of pSD for all 527 parameters of the input distribution with full dependence structure, are shown in figure 6 . Four points of the parameter space are considered, corresponding to the same configuration of marginal parameters described above, and $\rho_{i, j}=$ $\rho \in\{0,0.25,0.5,0.75\}$. For simplicity, we are only considering scenarios in which all pairwise correlation coefficients take the same value, but the methodology could encompass any point of interest in the parameter space. Observe that, when the independence assumption is dropped, not only the mean values, but also the standard deviations of the model inputs, become important. Perhaps the most interesting observation is that, even when $\rho=0$, the factor prioritization obtained from the full multivariate distribution differs from that of the reduced distribution of the independence case. In fact, the most important parameters in this case are $\mu_{12}, \mu_{31}, \sigma_{12}^{2}, \sigma_{31}^{2}$ and $\sigma_{12,31}^{2}$ (top graph). In other words, if the analyst relaxes the independence assumption at the epistemic uncertainty level, he should focus attention on properly estimating the mean and variance parameters of $X_{12}$ and $X_{31}$ and, more relevantly still, on ascertaining if the correlation between them is in fact null. Possible deviations from independence are most relevant with regards to possible dependence between inputs 12 and 31. For increasing values of $\rho$, correlation coefficients have a greater relevance. Regarding trend 


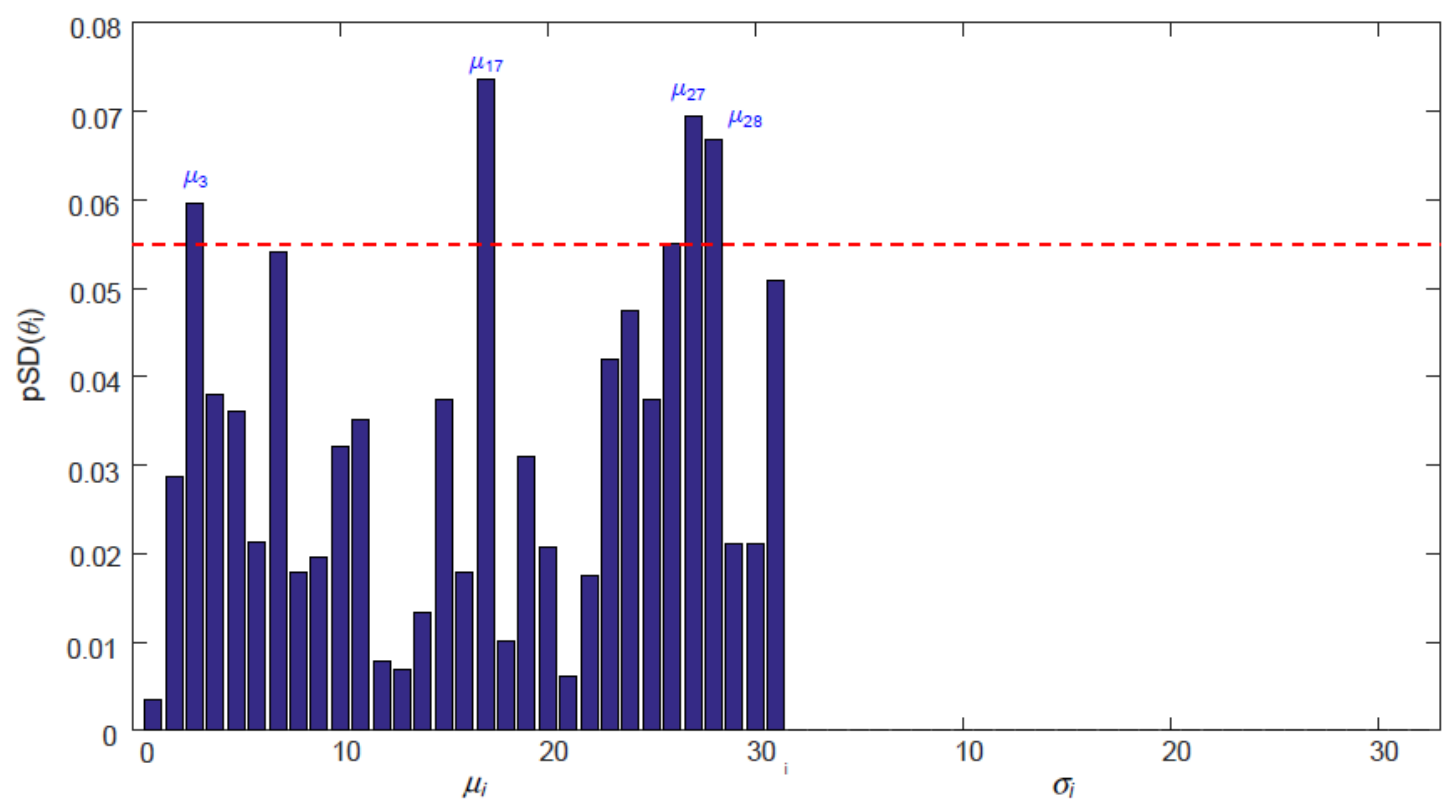

Fig. 4. $\widehat{\mathrm{pSD}}$ for the ATR reactor model, for parameters $\mu_{i}$ and $\sigma_{i}^{2}$ of the independent logNormal distributions for the 31 model inputs. The red dashed line indicates the $90 \%$ quantile, identifying the 4 most important parameters.
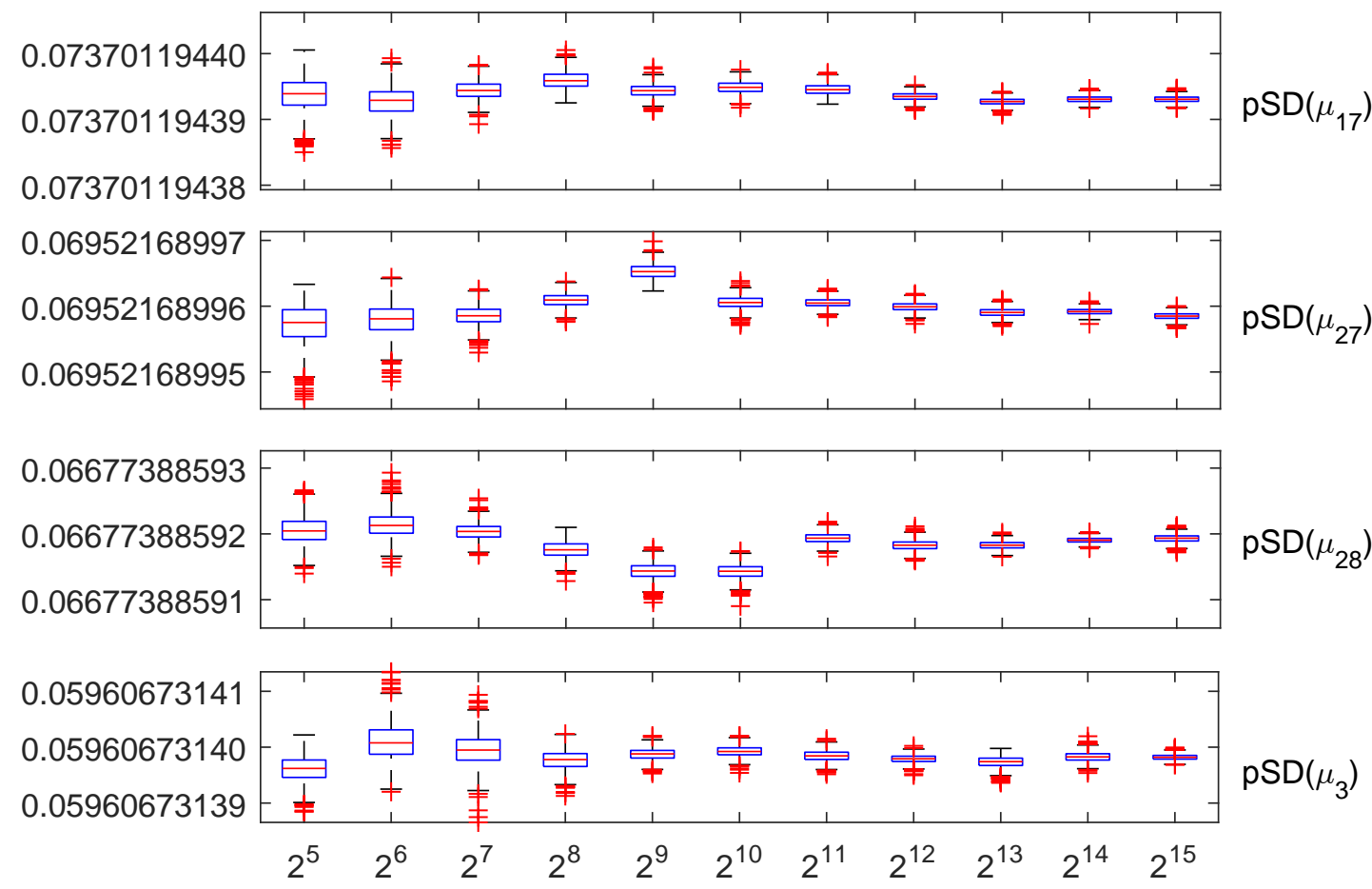

Fig. 5. Box plots of $\widehat{\mathrm{pSD}}$ of the 4 most important parameters, $\mu_{17}, \mu_{27}, \mu_{18}, \mu_{3}$, of the ATR reactor model, generated from $N_{B}=1,000$ bootstrap samples from datasets of increasing size, $N$ (represented on the horizontal axis). The box plots are nonoverlapping, and the ranking holds throughout. 

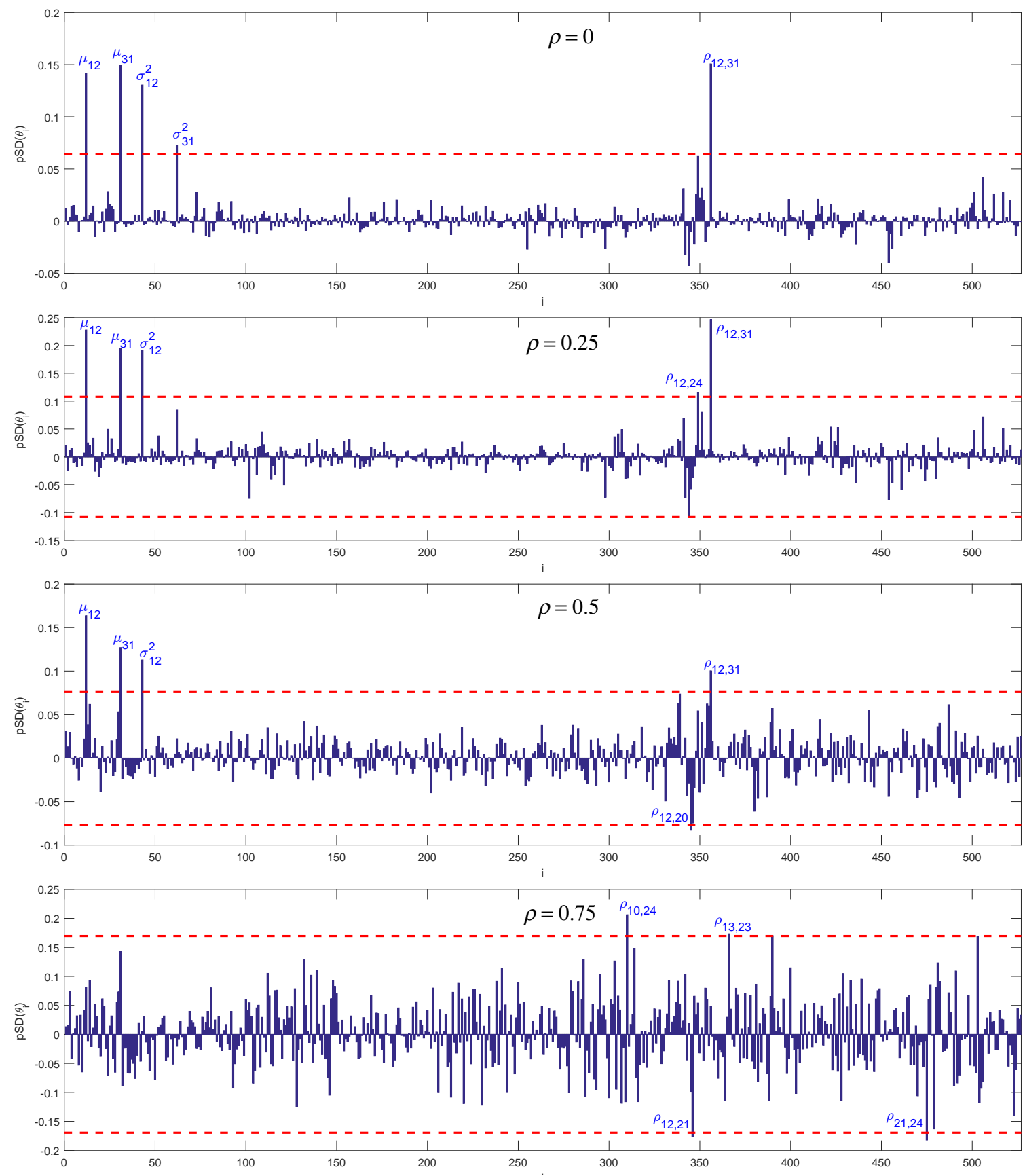

Fig. 6. $\widehat{\mathrm{pSD}}$ for the ATR reactor model, with respect to the 527 parameters of the full multivariate log-normal distribution: $\mu_{i}, \sigma_{i}^{2}, \rho_{i, j} ; i=1, \ldots, 31, i<j$. The parameter values and Monte Carlo sample size coincide with those of figure 4, with fixed correlation $\rho_{i, j}=\rho$ for $\rho \in\{0,0.25,0.5,0.75\}$ (from top to bottom). The dashed lines correspond to plus and minus $99 \%$ percentiles of $|\widehat{\mathrm{pSD}}|$, identifying roughly the 5 most important parameters.

identification, the sign of change is positive for some parameters and negative for others, even when $\rho=$ 0 , and the trend may change for different values of $\rho$. This contrasts with the results under the independence assumption, for which sign of change was always positive.

The method may also provide insights on the 
most important drivers at alternative modelling levels. Table I provides a summary.

At the parameter level, the importance of parameter groups may be considered. Under independence, the three most important distribution parameters are $\mu_{17}, \mu_{27}$ and $\mu_{28}$. Only means are relevant, while variances have practically no importance (correlations are absent from the probability distribution). In fact, column 2 of Table I, shows that the sum of all mean parameters (the group of parameters composed by all $\mu$ 's) is approximately equal to one. Under the full multivariate distribution, with zero correlation (the independence follows but is not a model assumption), the three most important parameters are $\rho_{12,31}, \mu_{31}$ and $\mu_{12}$. Observe that, now, the sum of the importance measures of all the mean parameters is (roughly) equal to 0.42 , that of the variance parameters is equal to 0.21 , and for correlation parameters it is 0.37 . When all correlation coefficients are fixed at $\rho=0.25$, the most important parameters are again $\rho_{12,31}, \mu_{12}$ and $\mu_{31}$, but with a slight change in order. The overall importances of the mean, variance and correlation parameters are now equal to $0.49,0.19$ and 0.32 , respectively. When $\rho=$ 0.50 or $\rho=0.75$, the correlation coefficients become the most important group of distribution parameters, with variances becoming more important than means when $\rho=0.75$. These results suggest that, while in the independence case the analyst only needs to focus on accurately estimating the $\mu_{i}$, when uncertainty about the correlation structure is taken into account, the analyst should also be concerned with accurate estimation of variances and correlation coefficients, with these becoming preponderant for high levels of dependence between model inputs.

Because of the additivity property of SD, the analyst can also identify the basic events whose parameters deserve more attention. In particular, under independence, the parameters associated with basic event 21 are the most important. When a dependence structure is introduced, the parameters of basic event 45 are the most important at all correlation levels. This is a reassuring result, because basic event 45 is, in fact, the initiating event of the sequence and has emerged as the most important basic event in previous studies. The parameters of basic events 16 and 29 follow as second and third most important, at the 0 and 0.25 correlation levels. These basic events refer to failures of the deepwell pumps in the firewater injection system (FIS). At the 0.50 and 0.75 correlation levels, the parameters of basic events 18 and 25 are the second and third most important groups. These basic events also refer to failure modes of the deepwell pumps.

We then consider the system level. Also following from additivity, the importance of each system is easily retrieved as a sum of the importance of the parameters of the basic events included in the system. There are two main systems, namely, the SCRAM system and the Firewater Injection System (Borgonovo et al., 2003). Table I shows that, for low levels of correlation, distribution parameters of the FIS system are more important than distribution parameters of the SCRAM system, while for correlation levels of 0.5 or 0.75 , the SCRAM parameters become more important than the FIS parameters. Interestingly, assuming independence (reduced set of distribution parameters) one assigns more importance to the FIS system, as opposed to the full model with zero correlation. This confirms the previous observations about the relevance of relaxing the independence assumption.

\section{CONCLUSIONS}

This work has addressed the sensitivity of the expected value of a risk metric to changes in the parameters of the distributions assigned to the model inputs. The proposed approach is based on the extension of the differential importance measure to a stochastic model output context. The resulting methodology has a series of practical advantages. It allows the analyst to overcome the limitations of using partial derivatives when parameters are denominated in different units. It allows to move between the parameter and the component levels straightforwardly. Towards application to realistic case studies, estimation has been considered in detail, providing a formal definition of two estimators that allow numerical approximation of the sensitivity measures from a single Monte Carlo sample, as in traditional uncertainty quantification. Additionally, the method provides a flexible tool for obtaining a quantitative assessment of the impact of assuming independence between model inputs in a risk analysis. A case study, in particular, indicates that the consequences can be notable, as allowing for correlations can lead to a drastic change in the ranking of the model input distribution parameters, as well as notable differences in the direction of change. Furthermore, it is possible to identify pairs of model inputs for which assessing dependence is more critical. 
Table I . The most important drivers at alternative modelling levels for the ATR-LLOCA sequence model

\begin{tabular}{|l|c|c|c|c|c|c|}
\hline $\begin{array}{l}\text { Distributional } \\
\text { assumption }\end{array}$ & $\begin{array}{c}\text { Importance of } \\
\mu_{1}-\mu_{31}\end{array}$ & $\begin{array}{c}\text { Importance of } \\
\sigma_{1}^{2}-\sigma_{31}^{2}\end{array}$ & $\begin{array}{c}\text { Importance of } \\
\rho_{1,2}-\rho_{30,31}\end{array}$ & $\begin{array}{c}\text { Most important } \\
\text { parameters }\end{array}$ & $\begin{array}{c}\text { Most important } \\
\text { basic events } \\
\text { important } \\
\text { system }\end{array}$ & $\begin{array}{c}\mu_{17}, \mu_{27}, \mu_{28} \\
\text { FIS }\end{array}$ \\
\hline Independence & 1 & 0 & 0 & 0.37 & $\rho_{12,31}, \mu_{31}, \mu_{12}$ & $45,16,29$ \\
\hline $\begin{array}{l}\text { Null correlation: } \\
\rho=0\end{array}$ & 0.42 & 0.21 & 0.32 & $\rho_{12,31}, \mu_{12}, \mu_{31}$ & $45,16,29$ \\
\hline $\begin{array}{l}\text { Low correlation: } \\
\rho=0.25\end{array}$ & 0.49 & 0.19 & 0.57 & $\mu_{12}, \mu_{31}, \sigma_{12}^{2}$ & $45,18,25$ \\
\hline $\begin{array}{l}\text { Medium } \\
\text { correlation: } \\
\rho=0.50\end{array}$ & 0.46 & -0.03 & $\rho_{10,24}, \rho_{21,24}$, & FCRAM \\
\hline $\begin{array}{l}\text { High correlation: } \\
\rho=0.75\end{array}$ & 0.46 & -0.75 & 1.29 & $\rho_{12,21}$ & $45,18,25$ \\
\hline
\end{tabular}

\section{ACKNOWLEDGMENT}

The authors wish to thank the editors Professor James Lambert and Professor Tony Cox for the editorial attention, and three anonymous reviewers for their many helpful comments.

\section{REFERENCES}

Anderson, B., Borgonovo, E., Galeotti, M., \& Roson, R. (2014). Uncertainty in Climate Change Modelling: Can Global Sensitivity Analysis be of Help? Risk Analysis, $34(2), 271-293$.

Apostolakis, G. (1990). The concept of probability in safety assessments of technological systems. Science, 250(4986), 1359-1364.

Apostolakis, G. (1994). A. Commentary on Model Uncertainty. In Proceedings of Workshop I in Advanced Topics in Risk and Reliability Analysis Model Uncertainty. Its Characterization and Quantification, October 20-22. 1993, (pp. 14-21).

Apostolakis, G. (2004). How Useful Is Quantitative Risk Assessment? Risk Analysis, 24 (3), 515-520.

Artzner, P., Delbaen, F., Eber, J., \& Heath, D. (1999). Coherent measures of risk. Mathematical Finance, 9, 203228 .

Aven, T. (2010). On the Need for Restricting the Probabilistic Analysis in Risk Assessments to Variability. Risk Analysis, 30(3), 354-360.

Aven, T. (2012). Foundational Issues in Risk Assessment and Risk Management. Risk Analysis, 32(10), 1647-1656.

Aven, T. (2016). Risk assessment and risk management: review of recent advances on their foundation. European Journal of Operational Research, 116(2), 235-248.

Aven, T. \& Zio, E. (2014). Foundational issues in risk assessment and risk management. Risk analysis, 34(7), $1164-72$.

Belles-Sampera, J., Guillen, M., \& Santolino, M. (2014). Beyond Value-at-Risk: GlueVaR Distortion Risk Measures. Risk Analysis, 34(1), 121-134.

Bier, V. (1983). A measure of uncertainty importance for components in fault trees. Transactions of the American Nuclear Society, 45(1), 384-5.

Borgonovo, E. (2006). Measuring uncertainty importance: investigation and comparison of alternative approaches. Risk analysis : an official publication of the Society for Risk Analysis, 26(5), 1349-1361.

Borgonovo, E. (2008). Sensitivity analysis of model output with input constraints: a generalized rationale for local methods. Risk analysis, 28(3), 667-680.

Borgonovo, E. \& Apostolakis, G. (2001). A new importance measure for risk-informed decision making. Reliability Engineering \& System Safety, 72(2), 193-212.

Borgonovo, E., Apostolakis, G., Tarantola, S., \& Saltelli, A. (2003). Comparison of global sensitivity analysis techniques and importance measures in PSA. Reliability Engineering E System Safety, 79(2), 175-185.

Borgonovo, E., Hazen, G., \& Plischke, E. (2016). A Common Rationale for Global Sensitivity Measures and their Estimation. Risk Analysis, 36(10), 1871-1895.

Borgonovo, E. \& Plischke, E. (2016). Sensitivity Analysis: A Review of Recent Advances. European Journal of Operational Research, 3(1), 869-887.

Borgonovo, E., Plischke, E., \& Morris, M. (2017). Functional Anova with Multiple Distributions: Implications for the Sensitivity Analysis of Computer Experiments. submitted. Borgonovo, E. \& Tarantola, S. (2008). Moment Independent and Variance-Based Sensitivity Analysis with Correlations. International Journal of Chemical Kinetics, 40(11), 687698.

Breeding, R., Helton, J., Gorham, E., \& Harper, F. (1992). Summary Description of the Methods Used in the Probabilistic Risk Assessments for NUREG-1150. Nuclear Engineering and Design 135:1-27. Nuclear Engineering and Design, 1-27.

Castillo, E., Gutierrez, J., \& Hadi, A. (1996). A new method for efficient symbolic propagation in discrete Bayesian networks. Networks, 28, 31-43.

Castillo, E., Gutierrez, J., \& Hadi, A. (1997). Sensitivity analysis in discrete Bayesian networks. IEEE Transactions on Systems, Man, and Cybernetics, 27, 412-423.

Confalonieri, R., Bellocchi, G., Tarantola, S., Acutis, M., Donatelli, M., \& Genovese, G. (2010). Sensitivity analysis of the rice model WARM in Europe: Exploring the effects of different locations, climates and methods of analysis on model sensitivity to crop parameters. Environmental Modelling and Software, 25(4), 479-488.

Davani, D. (1994). Parametric what-if analysis in MTTF: A single-run Monte-Carlo-based approach. Microelectronics Reliability, 34(2), 275-281.

Duintjer Tebbens, R., Pallansch, M., Kew, O., Sutter, R., Bruce Aylward, R., Watkins, M., Gary, H., Alexander, J., Jafari, H., Cochi, S., \& Thompson, K. (2008). Uncertainty and Sensitivity Analyses of a Decision Analytic Model for Posteradication Polio Risk Management. Risk Analysis, 28(4), 855-876.

Efron, B. \& Stein, C. (1981). The Jackknife Estimate of Variance. The Annals of Statistics, 9(3), 586-596.

Eschenbach, T. (1992). Spiderplots versus Tornado Diagrams for Sensitivity Analysis. Interfaces, 22(6), 40-46. 
Flage, R., Baraldi, P., Zio, E., \& Aven, T. (2013). Probability and possibility-based representations of uncertainty in fault tree analysis. Risk analysis, 33(1), 121-33.

Frey, H. \& Patil, S. (2002). Identification and review of sensitivity analysis methods. Risk Analysis, 22(3), 553578 .

Ghanem, R., Higdon, D., \& Owhadi, D. (Eds.). (2016). Handbook of Uncertainty Quantification. Springer.

Glasserman, P. \& Liu, Z. (2010). Sensitivity Estimates from Characteristic Functions. Operations Research, 58(6), 1611-1623.

Helton, J. (1993). Uncertainty and sensitivity analyses techniques for use in performance assessment for radioactive waste disposal. Reliability Engineering 8 System Safety, 42, 327-367.

Helton, J. \& Breeding, R. (1993). Calculation of reactor accident safety goals. Reliability Engineering and System Safety, 39, 129-158.

Helton, J. \& Davis, F. (2002). Illustration of Sampling-Based Methods for Uncertainty and Sensitivity Analysis. Risk Analysis, 22(3), 591-622.

Helton, J. \& Davis, F. (2003). Latin Hypercube Sampling and the Propagation of Uncertainty in Analyses of Complex Systems. Reliability Engineering \& System Safety, 81(1), 23-69.

Helton, J., Johnson, J., Sallaberry, C., \& Storlie, C. (2006). Survey of sampling-based methods for uncertainty and sensitivity analysis. Reliability Engineering $\&$ System Safety, 91(10-11), 1175-1209.

Helton, J. \& Oberkampf, W. (2004). Alternative representations of epistemic uncertainty. Reliability Engineering \& System Safety, 85(1-3), 1-10.

Helton, J. \& Sallaberry, C. (2012). Uncertainty and Sensitivity Analysis: From Regulatory Requirements to Conceptual Structure and Computational Implementation. IFIP Advances in Information and Communication Technology, 377, 60-76.

Hong, L. (2009). Estimating quantile sensitivities. Operations Research, 57(1), 118-130.

Howard, R. A. (1988). Decision Analysis: Practice and Promise. Management Science, 34(6), 679-695.

Iman, R. \& Helton, J. (1988). An investigation of uncertainty and sensitivity analysis techniques for computer models. Risk Analysis, 8, 71-90.

Iman, R. \& Hora, S. (1990). A robust measure of uncertainty importance for use in fault tree system analysis. Risk Analysis, 10, 401-406.

Iman, R., Johnson, M., \& Watson, C. (2005). Sensitivity Analysis for Computer Model Projections of Hurricane Losses. Risk Analysis, 25 (5), 1277-1298.

Kaplan, S. \& Garrick, B. (1981). On The Quantitative Definition of Risk. Risk Analysis, I(I), 11-27.

Kaplan, S., Haimes, Y., \& Garrick, B. (2001). Fitting Hierarchical Holographic Modeling into the Theory of Scenario Structuring and a Resulting Refinement to the Quantitative Definition of Risk. Risk Analysis, 21 (5), 807807.

Koks, E., Bočkarjova, M., de Moel, H., \& Aerts, J. (2015). Integrated direct and indirect flood risk modeling: Development and sensitivity analysis. Risk Analysis, 35(5), 882-900.

Lamboni, M., Iooss, B., Popelin, A.-L., \& Gamboa, F. (2013). Derivative-based Global Sensitivity Measures: General Links with Sobol' Indices and Numerical Tests. Mathematics and Computers in Simulation, 87, 45-54.

Li, J., Mosleh, A., \& Kang, R. (2011). Likelihood ratio gradient estimation for dynamic reliability applications. Reliability Engineering and System Safety, 96(12), 16671679.
Marrel, A., Iooss, B., Da Veiga, S., \& Ribatet, M. (2012). Global sensitivity analysis of stochastic computer models with joint metamodels. Statistics and Computing, 22(3), $833-847$.

North, W. (2010). Probability Theory and Consistent Reasoning. Risk Analysis, 30(3), 377-380.

North, W. (2011). Uncertainties, Precaution, and Science: Focus on the State of Knowledge and How It May Change. Risk Analysis, 31(10), 1526-1529.

Oakley, J. \& O'Hagan, A. (2004). Probabilistic Sensitivity Analysis of Complex Models: a Bayesian Approach. Journal of the Royal Statistical Society, Series B, 66(3), 751-769.

Paté-Cornell, M. (1996). Uncertainties in risk analysis: Six levels of Treatment. Reliability Engineering 85 System Safety, 54, 95-111.

Pedroni, N., Zio, E., \& Couplet, M. (2017). A Critical Discussion and Practical Recommendations on Some Issues Relevant to the Nonprobabilistic Treatment of Uncertainty in Engineering Risk Assessment. Risk Analysis, 37, 1315.1340.

Rakovec, O., Hill, M. C., Clark, M. P., Weerts, A. H., Teuling, A. J., \& Uijlenhoet, R. (2014). Distributed evaluation of local sensitivity analysis (DELSA), with application to hydrologic models. Water Resources Research, 50(1), 409426.

Nuclear Regulatory Commission (1975). Reactor safety study: An assessment of accident risks in U.S. commercial nuclear power plants. NUREG-75/014 (WASH-1400). Available from National Technical Information Service Springfield, Virginia 22161

Roustant, O., Fruth, J., Iooss, B., \& Kuhnt, S. (2014). Crossed-derivative Based Sensitivity Measures for Interaction Screening. Mathematics and Computers in Simulation, 105, 105-118.

Rubinstein, R. (1989). Sensitivity Analysis and Performance Extrapolation for Computer Simulation Models. Operations Research, 37(1), 72-82.

Rubinstein, R. \& Shapiro, A. (1993). R Y Rubinstein and A Shapiro. Discrete event systems: sensitivity analysis and stochastic optimization by the score function method. Wiley and Sons.

Saltelli, A. (2002). Sensitivity Analysis for Importance Assessment. Risk Analysis, 22(3), 579-590.

Saltelli, A., Ratto, M., Andres, T., Campolongo, F., Cariboni, J., Gatelli, D., Saisana, M., \& Tarantola, S. (2008). Global Sensitivity Analysis - The Primer. Chichester.

Saltelli, A., Ratto, M., Tarantola, S., \& Campolongo, F. (2005). Sensitivity Analysis for Chemical Models. Chemical Reviews, 105, 2811-2828.

Saltelli, A., Ratto, M., Tarantola, S., \& Campolongo, F. (2012). Update 1 of: Sensitivity Analysis for Chemical Models. Chemical Reviews, 112(5), 1-21.

Saltelli, A. \& Tarantola, S. (2002). On the Relative Importance of Input Factors in Mathematical Models: Safety Assessment for Nuclear Waste Disposal. Journal of the American Statistical Association, 97(459), 702-709.

Saltelli, A., Tarantola, S., \& Campolongo, F. (2000). Sensitivity Analysis as an Ingredient of Modelling. Statistical Science, 19(4), 377-395.

Saltelli, A., Tarantola, S., Campolongo, F., \& Ratto, M. (2004). Sensitivity Analysis in Practise - A Guide to Assessing Scientific Models. Chichester.

Samuelson, P. (1941). The Stability of Equilibrium: Comparative Statics and Dynamics. Econometrica, 9(2), 97-120.

Slutsky, E. (1925). Uber Stochastische Asymptoten und Grenzwerte. Metron, 5(3), 3-89.

Smith, A. \& Gans, W. (2015). Enhancing the Characterization of Epistemic Uncertainties in PM2.5 Risk Analyses. 
Risk Analysis, 35(3), 361-378.

Smith, C. (1998). Calculating conditional core damage probabilities for nuclear power plant operations Original Research Article. Reliability Engineering 83 System Safety, 59(3), 299-307.

Sobol', I. (1990). Estimation of the sensitivity of nonlinear mathematical models. Mat. Model., 2(1), 112-118.

Sobol', I. \& Kucherenko, S. (2009). Derivative Based Global Sensitivity Measures and their Links with Global Sensitivity Indices. Mathematics and Computers in Simulation, 79, 3009-3017.

Storlie, C., Swiler, L., Helton, J., \& Sallaberry, C. (2009). Implementation and evaluation of nonparametric regression procedures for sensitivity analysis of computationally demanding models. Reliability Engineering \& System Safety, 94 (11), 1735-1763.

Tsanakas, A. \& Millossovich, P. (2016). Sensitivity Analysis Using Risk Measures. Risk Analysis, 36(1), 30-48.

van der Gaag, L., Renooij, S., \& Coupe, V. (2007). Sensitivity Analysis of Probabilistic Networks. Advances in Probabilistic Graphical Models, Studies in Fuzziness and Soft Computing Volume 214, 2007, pp 103-124, 214, 103124.

Whitt, W. (2006). Sensitivity of performance in the ErlangA queueing model to changes in the model parameters. Operations Research, 54(2), 247-260.

\section{APPENDIX}

\section{Differential sensitivity measures and Taylor series}

Given a reference point $\mathbf{x}^{0}$, the change in model output $\Delta g=g(\mathbf{x})-g\left(\mathbf{x}^{0}\right)$ can be written as:

$$
\begin{array}{r}
\Delta g=\sum_{i=1}^{n} g_{i}^{\prime}\left(\mathbf{x}^{0}\right)\left(x_{i}-x_{i}^{0}\right)+\frac{1}{2} \sum_{i=1}^{n} \sum_{j=1}^{n} g_{i, j}^{\prime \prime}\left(x_{i}-x_{i}^{0}\right)\left(x_{j}-x_{j}^{0}\right) \\
+o\left(\left\|\left(\mathbf{x}-\mathbf{x}^{0}\right)^{2}\right\|\right) .
\end{array}
$$

If the input-output mapping, $g$, is smooth and the changes in the inputs are sufficiently small, omitting the higher order terms results in a negligible error. In this case, the first order approximation is denoted $\Delta g \simeq \sum_{j=1}^{n} g_{i}^{\prime}\left(\mathbf{x}^{0}\right)\left(x_{i}-x_{i}^{0}\right)=\sum_{j=1}^{n} g_{i}^{\prime}\left(\mathbf{x}^{0}\right) \mathrm{d} x_{i}$. The standard Leibnitz notation, $\mathrm{d} x_{i}=\left(x_{i}-x_{i}^{0}\right)$, is used to distinguish infinitesimal (sufficiently small) perturbations, from the (possibly larger) differences denoted by $\Delta x_{i}$.

Similarly, if $L(\boldsymbol{\theta})$ is differentiable and changes are small enough(Borgonovo \& Apostolakis, 2001), then it is possible to approximate $\Delta L \simeq \sum_{i=1}^{n} L_{i}^{\prime}\left(\boldsymbol{\theta}^{0}\right)\left(\theta_{i}-\right.$ $\left.\theta_{i}^{0}\right)$, and equation (11) can be rewritten in terms of Taylor expansion, as

$$
\mathrm{SD}_{j}=\frac{\Delta_{j} L}{\Delta L}=\frac{L_{j}^{\prime}\left(\boldsymbol{\theta}^{0}\right)\left(\theta_{j}-\theta_{j}^{0}\right)}{\sum_{i=1}^{n} L_{i}^{\prime}\left(\boldsymbol{\theta}^{0}\right)\left(\theta_{i}-\theta_{i}^{0}\right)} .
$$

\section{Calculations for multivariate normal (and log-normal) input distribution}

It is simple to see that, under proportional perturbations, for the model considered in section 5 ,

$$
\begin{aligned}
& \operatorname{pSD}_{\mu_{1}}=\frac{1}{C} \mu_{2} \quad \operatorname{pSD}_{\mu_{2}}=\frac{1}{C} \mu_{1} \\
& \mathrm{pSD}_{\mu_{3}}=\frac{1}{C} \\
& \mathrm{pSD}_{\sigma_{1}^{2}}=\frac{1}{C} \frac{\rho_{1,2} \sigma_{2}}{2 \sigma_{1}} \quad \operatorname{pSD}_{\sigma_{2}^{2}}=\frac{1}{C} \frac{\rho_{1,2} \sigma_{1}}{2 \sigma_{2}} \quad \operatorname{pSD}_{\sigma_{3}^{2}}=0 \\
& \operatorname{pSD}_{\rho_{1,2}}=\frac{1}{C} \sigma_{1} \sigma_{2} \quad \operatorname{pSD}_{\rho_{1,3}}=0 \quad \operatorname{pSD}_{\rho_{2,3}}=0 \text {, }
\end{aligned}
$$

where

$$
C=\mu_{2}+\mu_{1}+\frac{\rho_{1,2} \sigma_{2}}{2 \sigma_{1}}+\frac{\rho_{1,2} \sigma_{1}}{2 \sigma_{2}}+\sigma_{1} \sigma_{2} .
$$

However, for more complex models, direct calculations may not be possible. In this case, SD may be evaluated using the score function, as in equation (7). When the input distribution is Multivariate Normal, its pdf is given by

$$
f(\mathbf{x} ; \mu, \Sigma)=\frac{1}{\sqrt{(2 \pi)^{3}|\Sigma|}} \exp \left\{-\frac{1}{2}(\mathbf{x}-\mu)^{\prime} \Sigma^{-1}(\mathbf{x}-\mu)\right\},
$$

where $|\Sigma|$ denotes the determinant of the covariance matrix $\Sigma$. Therefore, the log-likelihood function is

$\log f(\mathbf{x} ; \mu, \Sigma)=-\frac{3}{2} \log (2 \pi)-\log (|\Sigma|)-\frac{1}{2}(\mathbf{x}-\mu)^{\prime} \Sigma^{-1}(\mathbf{x}-\mu)$.

The score function, in matrix form, can be easily obtained by differentiation:

$$
\begin{gathered}
S(\mu ; \mathbf{X})=\frac{\partial \log f(\mathbf{x} ; \mu, \Sigma)}{\partial \mu}=\Sigma^{-1}(\mathbf{x}-\mu) \\
S(\Sigma ; \mathbf{X})=\frac{\partial \log f(\mathbf{x} ; \mu, \Sigma)}{\partial \Sigma} \\
=-\frac{1}{2}\left[\Sigma^{-1}-\Sigma^{-1}(\mathbf{x}-\mu)(\mathbf{x}-\mu)^{\prime} \Sigma^{-1}\right] .
\end{gathered}
$$

Since $\sigma_{i, i}=\sigma_{i}^{2}$, clearly the $i$-th element in the diagonal of this matrix corresponds to $S\left(\sigma_{i}^{2} ; \mathbf{X}\right)$. On the other hand, $\sigma_{i, j}=\rho_{i, j} \sigma_{i} \sigma_{j}$, so in order to obtain $S\left(\rho_{i, j} ; \mathbf{X}\right)$, the entry $(i, j)$ of the matrix must be multiplied by $\sigma_{i}, \sigma_{j}$ (by the chain rule). It is, therefore, possible to construct a Monte Carlo estimator of pSD for each distribution parameter through the Score function method, by generating samples from the corresponding multivariate normal distribution with the desired parameter values (see equations 17 and 18).

No additional calculations are required when the input distribution is log-normal. The expression of the score function for a multivariate log-normal distribution with correlations is cumbersome, as well as the calculation of the corresponding score function. However, one can use the property that a log-normal random variable is the exponential of a normal random variable to simplify calculations. 
Indeed, let $\mathbf{X}=\left(X_{1}, \ldots, X_{n}\right)$ follow a multivariate log-normal distribution with probability density function

$$
\begin{aligned}
f_{\mathbf{X}}(\mathbf{x} \mid \mu, \Sigma) & =(2 \pi)^{-d / 2}\left[\prod_{i=1}^{d} \frac{1}{x_{i}}\right]|\Sigma|^{-1 / 2} \\
& \times \exp \left\{-\frac{1}{2}(\ln \mathbf{x}-\mu)^{T} \Sigma^{-1}(\ln \mathbf{x}-\mu)\right\},
\end{aligned}
$$

where $\ln \mathbf{x}=\left(\ln x_{1}, \ldots, \ln x_{n}\right)$. In this case, the marginal distribution of each $X_{i}$ is log-normal, with probability density function given by

$$
f_{X_{i}}\left(x_{i} \mid \mu_{i}, \sigma_{i}^{2}\right)=\frac{1}{x_{i} \sqrt{2 \pi \sigma_{i}^{2}}} \exp \left\{-\frac{\left(\log x_{i}-\mu_{i}\right)^{2}}{2 \sigma_{i}^{2}}\right\} \text {. }
$$

If the covariance matrix is not diagonal, a dependence structure is present, with

$$
\operatorname{Cov}\left(X_{i}, X_{j}\right)=\left(\exp \left\{\sigma_{i, j}\right\}-1\right) \exp \left\{\mu_{i}+\mu_{j}+\frac{\sigma_{i, i}+\sigma_{j, j}}{2}\right\},
$$

where $\sigma_{i, i}=\rho_{i, j} \sigma_{i} \sigma_{j}$ for $i \neq j$.

In this case, $\mathbf{W}=\left(\ln X_{1}, \ldots, \ln X_{d}\right)$ follows a $d$-variate normal distribution with mean $\mu$ and covariance matrix $\Sigma$. Rather than finding convoluted expressions for the score function of $\mathbf{X}$, we may write $g(\mathbf{X})=g(\exp (\mathbf{W}))$. Since the parameters coincide, it is possible to estimate the sensitivity measures through the score function of the multivariate Normal distribution. 